\title{
Superspace formulation and correlation functions of $3 d$ superconformal field theories
}

\author{
Amin A. Nizami, ${ }^{a}$ Tarun Sharma $^{b}$ and V. Umesh ${ }^{b}$ \\ ${ }^{a}$ DAMTP, Centre for Mathematical Sciences, \\ Wilberforce Road, Cambridge CB3 0WA, U.K. \\ ${ }^{b}$ Department of Theoretical Physics, Tata Institute of Fundamental Research, \\ Homi Bhabha Road, Colaba-400005, India \\ E-mail: A.A.Nizami@damtp.cam.ac.uk, tarun@theory.tifr.res.in, \\ umesh@theory.tifr.res.in
}

ABSTRACT: We study $3 d$ SCFTs in the superspace formalism and discuss superfields and on-shell higher spin current multiplets in free $3 d$ SCFTs with $\mathcal{N}=1,2,3,4$ and 6 superconformal symmetry. For $\mathcal{N}=13 \mathrm{~d}$ SCFTs we determine the superconformal invariants in superspace needed for constructing 3-point functions of higher spin operators, find the non-linear relations between the invariants and consequently write down all the independent invariant structures, both parity even and odd, for various 3-point functions of higher spin operators.

Keywords: Field Theories in Lower Dimensions, Superspaces, Conformal and W Symmetry, Chern-Simons Theories

ARXIV EPRINT: 1308.4778 


\section{Contents}

1 Introduction 1

$\begin{array}{llr}2 & \text { Superspace } & 2\end{array}$

3 Free superconformal theories in superspace 5

$3.1 \mathcal{N}=1 \quad 6$

$\begin{array}{ll}3.2 \mathcal{N}=2 & 6\end{array}$

$3.3 \mathcal{N}=3 \quad 8$

$3.4 \mathcal{N}=4 \quad 9$

$\begin{array}{lr}3.5 \mathcal{N}=6 & 10\end{array}$

4 Currents $\quad 11$

4.1 General structure of the current superfield 11

4.2 Free field construction of currents 13

$\begin{array}{ll}4.2 .1 \mathcal{N}=1 & 13\end{array}$

$\begin{array}{lll}4.2 .2 \mathcal{N}=2 & 13\end{array}$

$\begin{array}{lll}4.2 .3 \mathcal{N}=3 & 14\end{array}$

$\begin{array}{lll}4.2 .4 \mathcal{N}=4 & 14\end{array}$

$\begin{array}{ll}4.2 .5 \mathcal{N}=6 & 15\end{array}$

5 Weakly broken conservation $\quad 16$

$\begin{array}{lll}6 & \text { Two-point functions } & 17\end{array}$

7 Three-point functions $\quad 19$

7.1 Superconformal invariants for three-point functions of $\mathcal{N}=1$ higher spin operators 20

7.1.1 Construction of the parity odd fermionic invariant $T \quad 20$

$\begin{array}{lll}7.2 & \text { Relations between the invariant structures } & 23\end{array}$

$\begin{array}{lll}\text { 7.3 Simple examples of three point functions } & 25\end{array}$

7.3.1 Independent invariant structures for three point functions 25

$\begin{array}{ll}\text { 7.3.2 Three point functions of conserved currents } & 28\end{array}$

8 Summary and outlook $\quad 30$

A Conventions $\quad 31$

A.1 Spacetime spinors 31

A.2 $R$-symmetry $\quad 32$

$\begin{array}{lll}\text { A.2.1 } & \mathrm{SO}(3) & 32\end{array}$

$\begin{array}{lll}\text { A.2.2 } & \mathrm{SO}(4) & 33\end{array}$

$\begin{array}{lll}\text { A.2.3 } & \mathrm{SO}(6) & 33\end{array}$ 
C Superconformal spectrum of $\mathcal{N}=1,2,3,4,6$ theories $\quad 36$

$\begin{array}{lll}\text { C. } 1 \mathcal{N}=1 & 36\end{array}$

$\begin{array}{ll}\text { C. } 2 \mathcal{N}=2 & 36\end{array}$

$\begin{array}{lll}\text { C.3 } \mathcal{N}=3 & 37\end{array}$

$\begin{array}{ll}\text { C. } 4 \mathcal{N}=4 & 37\end{array}$

$\begin{array}{ll}\text { C.5 } \mathcal{N}=6 & 38\end{array}$

\section{Introduction}

It has recently been realized that non-abelian Chern-Simons theories coupled to fundamental matter fields in 3 dimensions are exactly solvable in the large $N$ limit. These theories have an interesting 'current algebra' structure involving almost conserved higher spin fields, appear to enjoy invariance under nontrivial level-rank type strong-weak coupling dualities, and also appear to admit a bulk dual description in terms of Vasiliev's equations for higher spin fields [1-16].

The new results obtained for large $N$ vector Chern-Simons theories are exciting partly because they apply to non-supersymmetric theories. Most of the results obtained in $[7,17]$, however, have simple extensions to the supersymmetric counterparts of the theories studied there (see e.g. [8]). For instance, it should be possible to extend the results of Maldacena and Zhiboedov [3,4] to obtain the exact form of the higher spin current algebra and the correlation functions of supersymmetric Chern-Simons coupled to fundamental matter fields with minimal matter content.

In order to extend recent results in the study of matter Chern-Simons theories to their supersymmetric counterparts, it would be convenient to have a formulation of these theories in superspace. Offshell superspace formulations of theories with extended supersymmetry are complicated and very messy. Moreover the abstract study of supersymmetric matter Chern Simons theories, along the lines of [3, 4], does not need an offshell formalism. In this paper we initiate the development of onshell superspace techniques to study superconformal field theories. In particular we present a detailed study of free superconformal field theories in superspace using onshell techniques. We present a superspace construction of higher spin supercurrents in free theories, and describe the structural form of the current algebra of the corresponding higher spin currents once we include the effect of interactions. We also study the correlation functions of higher spin currents in superspace; in particular we conjecture that superconformal invariance and current conservation constrains the form of the three point functions of higher spin currents to a linear combination of the unique 'free' structure and a parity odd structure; we present evidence in favor of this conjecture.

This paper is structured as follows. In section 2 we consider $3 \mathrm{~d}$ superspace, and the differential form of various operators which act in it. The construction of superconformally 
covariant structures in superspace is reviewed. Section 3 deals with specifics of $\mathcal{N}=$ $1,2,3,4$ and 6 superconformal symmetry in superspace and the construction of superfield multiplets. In section 4 on-shell supercurrent multiplets for higher spin currents in the free theory are constructed out of the superfields. In section 5 we make a few remarks about the structure of anomalous conservation equations for 3d CFTs and SCFTs with weakly broken higher spin symmetry. In sections 6 and 7, which are essentially independent of sections 3,4 and 5 and can be read independently, we turn to correlation functions of $\mathcal{N}=13 \mathrm{~d}$ SCFTs. In section 6 we give the form of the 2-point function of a spin $s$ operator and give an elementary derivation, on the basis of symmetry and dimensional arguments, of the 2-point function of two spin half operators and explicitly compute a 2-point correlator in the free theory. In section 7 we turn to 3-point correlation functions - we first construct parity even and odd superconformal invariants in superspace, determine the myriad nonlinear relations between them and then use these results (in section 7.3) to determine the independent invariant structures which can arise in various 3-point functions of higher spin operators. This section is essentially an extension, to the superconformal case, of many of the results of [17]. We build the invariants using the superconformal covariant structures constructed by J-H Park and H. Osborn [18-22] augmented by the polarization spinor formalism used by [17]. In appendix A we list our conventions and some useful identities. In appendix B we give single trace conformal primary decomposition of a free $\mathrm{U}(N)$ theory of a single complex scalar and complex fermion. In appendix $\mathrm{C}$ we present the full single trace superconformal primary spectrum of the theories discussed in section 3 .

\section{Superspace}

We begin by reviewing superspace in three dimensions and the covariant structures that it admits, relying heavily on the paper of Park [21]. Our conventions are summarized in appendix A.

In order to study $\mathcal{N}=m$ superconformal field theories in 3 dimensions we employ a superspace whose coordinates are the 3 spacetime coordinates $x^{\mu}$ together with the $2 m$ fermionic coordinates $\theta_{\alpha}^{a}$. Here $\alpha=1,2$ is a spacetime spinor index while $a=1 \ldots m$ is the $R$-symmetry index, where the $\theta \mathrm{s}$ (and the supercharges $Q_{\alpha}^{a} \mathrm{~s}$ ) are Majorana spinors that lie in the vector representation of the $R$-symmetry group $\operatorname{SO}(\mathcal{N})$. The superconformal algebra, listed in (A.7) in appendix A.1, is implemented in superspace by the construction

$$
\begin{aligned}
P_{\mu} & =-i \partial_{\mu} \\
M_{\mu \nu} & =-i\left(x_{\mu} \partial_{\nu}-x_{\nu} \partial_{\mu}-\frac{1}{2} \epsilon_{\mu \nu \rho}\left(\gamma^{\rho}\right)_{\alpha}^{\beta} \theta_{\beta}^{a} \frac{\partial}{\partial \theta_{\alpha}^{a}}\right)+\mathcal{M}_{\mu \nu}, \\
D & =-i\left(x^{\nu} \partial_{\nu}+\frac{1}{2} \theta^{\alpha a} \frac{\partial}{\partial \theta_{a}^{\alpha}}\right)+\Delta \\
K_{\mu} & =-i\left(\left(x^{2}+\frac{\left(\theta^{a} \theta^{a}\right)^{2}}{16}\right) \partial_{\mu}-2 x_{\mu}\left(x \cdot \partial+\theta^{\alpha a} \frac{\partial}{\partial \theta_{a}^{\alpha}}\right)+\left(\theta^{a} X_{+} \gamma_{\mu}\right)^{\beta} \frac{\partial}{\partial \theta_{a}^{\beta}}\right) \\
& =x^{\nu} M_{\nu \mu}-x_{\mu} D+\frac{i}{2}\left(\theta^{a} \gamma_{\mu} X\right)^{\alpha} \frac{\partial}{\partial \theta_{a}^{\alpha}}-\frac{i}{16}\left(\theta^{a} \theta^{a}\right)^{2} \partial_{\mu}+\frac{\left(\theta^{a} \theta^{a}\right)}{4}\left(\theta^{b} \gamma_{\mu}\right)^{\alpha} \frac{\partial}{\partial \theta_{b}^{\alpha}}
\end{aligned}
$$




$$
\begin{aligned}
Q_{\alpha}^{a} & =\frac{\partial}{\partial \theta_{a}^{\alpha}}-\frac{i}{2} \theta^{\beta a}\left(\gamma^{\mu}\right)_{\beta \alpha} \partial_{\mu}, \\
S_{\alpha}^{a} & =-\left(X_{+}\right)_{\alpha}^{\beta} Q_{\beta}^{a}-i \theta^{a} \theta^{b} \frac{\partial}{\partial \theta_{b}^{\alpha}}-i \theta_{\alpha}^{a} \theta^{b \beta} \frac{\partial}{\partial \theta_{b}^{\beta}}+\frac{i}{2}\left(\theta^{b} \theta^{b}\right) \frac{\partial}{\partial \theta_{a}^{\alpha}} \\
& =-\left(X_{-}\right)_{\alpha}^{\beta} \frac{\partial}{\partial \theta_{a}^{\beta}}+\frac{\theta_{\alpha}^{a}}{2} D+\frac{1}{4} \epsilon_{\mu \nu \rho}\left(\gamma^{\rho} \theta^{a}\right)_{\alpha} M^{\mu \nu}-\frac{\left(\theta^{b} \theta^{b}\right)}{8} \theta^{a \beta} \partial_{\beta \alpha}-\frac{3 i}{4}\left(\theta_{\alpha}^{a} \theta \frac{\partial}{\partial \theta}+\theta^{a} \theta^{b} \frac{\partial}{\partial \theta_{b}^{\alpha}}\right), \\
I^{a b} & =-i\left(\theta^{a} \frac{\partial}{\partial \theta_{b}}-\theta^{b} \frac{\partial}{\partial \theta_{a}}\right)+\mathcal{I}^{a b} .
\end{aligned}
$$

Here the derivative expressions act on superspace coordinates while the operators $\mathcal{M}, \Delta$ and $\mathcal{I}^{a b}$ act on the operators (states) which carry tensor structure, non-zero scaling dimensions and transform non-trivially under $R$-symmetry. All indices are contracted in matrix notation (the spinors are contracted from north-west to south-east, see appendix A.1) and the definitions of $X_{+}, X_{-}$are given in (2.9). Note that $x^{2}+\frac{\left(\theta^{a} \theta^{a}\right)^{2}}{16}=\frac{1}{2}\left(X_{+} X_{-}\right)_{\alpha}^{\alpha}$ (this combination appears in the expression for $K_{\mu}$ above). Below we will often have occasion to use a 'supersymmetric' derivative operator $D_{\alpha}^{a}$ defined by

$$
D_{\alpha}^{a}=\frac{\partial}{\partial \theta_{a}^{\alpha}}+\frac{i}{2} \theta^{a \beta} \partial_{\beta \alpha},
$$

The operator $D_{\alpha}^{i}$ has the property that it anticommutes will all supersymmetry generators

$$
\left\{D_{\alpha}^{a}, Q_{\beta}^{b}\right\}=0
$$

Note also that

$$
\left\{D_{\alpha}^{a}, D_{\beta}^{b}\right\}=-P_{\alpha \beta} \delta^{a b}
$$

In the sequel we will sometimes require to construct functions built out of coordinates in superspace that are invariant under superconformal transformations. Given two points in superspace, $\left(x_{1}, \theta_{1}\right)$ and $\left(x_{2}, \theta_{2}\right)$, it is obvious that $\theta_{12}=\theta_{1}-\theta_{2}$ is annihilated by the supersymmetry generators. It is also easy to verify that the supersymmetrized coordinate difference

$$
\tilde{x}_{12}^{\mu}=x_{12}^{\mu}+\frac{i}{2} \theta_{1}^{a \alpha}\left(\gamma^{\mu}\right)_{\alpha}^{\beta} \theta_{2 \beta}^{a}
$$

is also annihilated by all $Q_{\alpha}$.

Any vector of $\mathrm{SO}(2,1)$ may equally be regarded as a symmetrized bispinor. So $x^{\mu}$ may be represented in terms of bispinors by the $2 \times 2$ matrix $X=x \cdot \gamma$. In this notation (2.5) may be rewritten as

$$
\left(\tilde{X}_{12}\right)_{\alpha}^{\beta}=\left(X_{12}\right)_{\alpha}^{\beta}+i \theta_{1 \alpha}^{a} \theta_{2}^{a \beta}+\frac{i}{2}\left(\theta_{1}^{a} \theta_{2}^{a}\right) \delta_{\alpha}^{\beta}
$$

While an arbitrary function of $\theta_{12}$ and $\tilde{X}_{12}$ is annihilated by the supersymmetry operator, it is not, in general, annihilated by the generator of superconformal transformations. In order to build superconformally invariant expressions it is useful to note that

$$
S_{\alpha}^{a}=I Q_{\alpha}^{a} I
$$


where $I$ is the superinversion operator, whose action on the coordinates of superspace is given by

$$
I\left(x^{\mu}\right)=\frac{x^{\mu}}{x^{2}+\frac{\left(\theta^{a} \theta^{a}\right)^{2}}{16}}
$$

To define the superinversion properties of spinors, it is useful to define the objects

$$
X_{ \pm}=X \pm \frac{i}{4}\left(\theta^{a} \theta^{a}\right) \mathbb{1}
$$

It follows from (2.8) that this object transforms homogeneously under inversions

$$
\begin{aligned}
I\left(X_{ \pm}\right) & =X_{ \pm}^{-1} \\
I\left(\theta_{\alpha}^{a}\right) & =\left(X_{+}^{-1} \theta^{a}\right)_{\alpha} \\
I\left(\theta^{a \beta}\right) & =-\left(\theta^{a} X_{-}^{-1}\right)^{\beta}
\end{aligned}
$$

(Here $X$ is the $2 \times 2$ matrix corresponding to a particular superspace point, not a coordinate difference).

Using these rules it follows that the following objects (see e.g. [18-22] transform homogeneously under inversions:

$$
\begin{aligned}
& \left(X_{i j+}\right)_{\alpha}^{\beta}=\left(X_{i+}\right)_{\alpha}^{\beta}-\left(X_{j-}\right)_{\alpha}^{\beta}+i \theta_{i \alpha}^{a} \theta_{j}^{\beta a} \\
& \left(X_{i j-}\right)_{\alpha}^{\beta}=\left(X_{i-}\right)_{\alpha}^{\beta}-\left(X_{j+}\right)_{\alpha}^{\beta}-i \theta_{j \alpha}^{a} \theta_{i}^{\beta a}
\end{aligned}
$$

For example,

$$
I\left(X_{i j+}\right)_{\alpha}^{\beta}=I\left(\left(X_{i+}\right)_{\alpha}^{\beta}-\left(X_{j-}\right)_{\alpha}^{\beta}+i \theta_{i \alpha}^{a} \theta_{j}^{a \beta}\right)=-\left(X_{i+}^{-1}\right)_{\alpha}^{\gamma}\left(X_{i j+}\right)_{\gamma}^{\delta}\left(X_{j-}^{-1}\right)_{\delta}^{\beta}
$$

Moreover it may be demonstrated [18-22] that

$$
X_{i j \pm}=\tilde{X}_{i j} \pm \frac{i}{4} \theta_{i j}^{2} \mathbb{1}
$$

In other words $X_{i j \pm}$ transform homogeneously under inversions and are also annihilated by the generators of supersymmetry. In performing various manipulations it is useful to note that

$$
\begin{aligned}
X_{+} X_{-} & =\left(x^{2}+\frac{1}{16}\left(\theta^{a} \theta^{a}\right)^{2}\right) \mathbb{1} \\
X_{i j+} X_{i j-} & =\left(\tilde{x}_{i j}^{2}+\frac{1}{16}\left(\theta_{i j}^{a} \theta_{i j}^{a}\right)^{2}\right) \mathbb{1}
\end{aligned}
$$

so that

$$
\begin{aligned}
\left(X_{ \pm}\right)^{-1} & =\frac{X_{\mp}}{x^{2}+\frac{1}{16}\left(\theta^{a} \theta^{a}\right)^{2}} \\
\left(X_{i j \pm}\right)^{-1} & =\frac{X_{i j \mp}}{\tilde{x}_{i j}^{2}+\frac{1}{16}\left(\theta_{i j}^{a} \theta_{i j}^{a}\right)^{2}}
\end{aligned}
$$

(note that the the $R$-symmetry index $a$ is summed over but that, throughout, $i, j(=1,2,3)$ label points in superspace and are not summed over). 
There also exist fermionic covariant structures (which are identically zero in the nonsupersymmetric case) which are constructed out of the superspace co-ordinates as follows [18-22]:

$$
\Theta_{1 \alpha}^{a}=\left(\left(X_{21+}^{-1} \theta_{21}^{a}\right)_{\alpha}-\left(X_{31+}^{-1} \theta_{31}^{a}\right)_{\alpha}\right)
$$

$\Theta_{2}, \Theta_{3}$ are defined similarly. Its transformation properties under superinversion are

$$
\Theta_{i \alpha}^{a} \rightarrow-\left(X_{i-}\right)_{\alpha}^{\beta} \Theta_{i \beta}^{b} I_{b}^{a} \quad \Theta_{i}^{\alpha a} \rightarrow I_{b}^{T a} \Theta_{i}^{\beta b}\left(X_{i+}\right)_{\beta}^{\alpha}
$$

The basic covariant structures $X_{i j \pm}, \Theta_{i \alpha}^{a}$ are annihilated by the generators of supersymmetry. For this reason they form the basic building blocks for the construction of superconformal invariants, as we will explain in a later section.

Polarization spinors: since we will be dealing extensively with higher spin operators and their correlators, it will be useful to adopt a formalism, developed in [17], ${ }^{1}$ in which the information about the tensor structure is encoded in polarization spinors: $\lambda_{\alpha}$. These auxiliary objects are book-keeping devices to keep track of the tensorial nature of correlators in an efficient manner. They are defined to be real, bosonic, two-component objects transforming as spinors of the $3 d$ Lorentz group (see [17]). Being spinors in $2+1$ dimensions fixes their transformation law under superinversions:

$$
\lambda_{\alpha} \rightarrow\left(X_{+}^{-1} \lambda\right)_{\alpha}, \quad \lambda^{\beta} \rightarrow-\left(\lambda X_{-}^{-1}\right)^{\beta}
$$

(This is the same as the transformation law of the $\theta$ 's).

A higher spin primary operator $J_{\mu_{1} \mu_{2} \ldots \ldots \mu_{s_{i}}}$ with spin $s_{i}$ can be represented in spinor components by $J_{\alpha_{1} \alpha_{2} \ldots \ldots \alpha_{2 s_{i}}} \equiv\left(\sigma^{\mu_{1}}\right)_{\alpha_{1} \alpha_{2}}\left(\sigma^{\mu_{2}}\right)_{\alpha_{3} \alpha_{4}} \ldots\left(\sigma^{\mu_{s}}\right)_{\alpha_{2 s-1} \alpha_{2 s}} J_{\mu_{1} \mu_{2} \ldots \ldots \mu_{s_{i}}}$. We note that this represents an operator supermultiplet in contradistinction to [17] where the nonsupersymmetric conformal case was considered (also, $J$ need not necessarily be a conserved current). We then define $J_{s_{i}} \equiv \lambda^{\alpha_{1}} \lambda^{\alpha_{2}} \ldots \lambda^{\alpha_{2 s_{i}}} J_{\alpha_{1} \alpha_{2} \ldots \ldots \alpha_{2 s_{i}}}$.

The 3-point function $\left\langle J_{s_{1}}\left(x_{1}, \theta_{1}, \lambda_{1}\right) J_{s_{2}}\left(x_{2}, \theta_{2}, \lambda_{2}\right) J_{s_{3}}\left(x_{3}, \theta_{3}, \lambda_{3}\right)\right\rangle$ is then a superconformal invariant constructed out of three points in (augmented) superspace with co-ordinates labeled by $\left(x_{i}, \theta_{i}, \lambda_{i}\right)$. The tensor structure of the correlator, instead of being represented by indices, is encoded by the polynomial in $\lambda$ 's (the 3 -point function being a multinomial with degree $\lambda_{1}^{2 s_{1}} \lambda_{2}^{2 s_{2}} \lambda_{3}^{2 s_{3}}$ for each term).

\section{Free superconformal theories in superspace ${ }^{2}$}

In this section we study free superconformal theories, with $\mathcal{N}=1,2,3,4$ and 6 supersymmetry in superspace. ${ }^{3}$

\footnotetext{
${ }^{1}$ See also [23] for a similar approach.

${ }^{2}$ In this paper we deal exclusively with onshell superspace. For offshell $3 \mathrm{~d}$ superspace and multiplets in theories with and without gravity, see [24, 25].

${ }^{3}$ Sections 3, 4 and 5 have been worked out in collaboration with Shiraz Minwalla.
} 


\section{$3.1 \mathcal{N}=1$}

$\mathcal{N}=1$ superspace consists of points $z^{A}=\left(x^{\mu}, \theta_{\alpha}\right)$, where $\theta_{\alpha}$ is a Majorana spinor. There are two real supercharges $Q_{\alpha}$; these operators are implemented in superspace by the expressions (2.1) with $\mathcal{N}=1$.

The 'minimal' free $\mathcal{N}=1$ theory consists of a single complex scalar field together with a single complex fermion. These fields are packaged together into a single complex $\mathcal{N}=1$ superfield $\Phi$ subject to the supersymmetric equation of motion

$$
D^{\alpha} D_{\alpha} \Phi=0
$$

Note that $\Phi$, like any scalar $\mathcal{N}=1$ superfield, may be expanded in components as

$$
\begin{aligned}
& \Phi=\phi+\theta \psi+\frac{\theta^{2}}{2} F \\
& \bar{\Phi}=\bar{\phi}-\theta \psi^{*}-\frac{\theta^{2}}{2} \bar{F} .
\end{aligned}
$$

By expanding (3.1) in components it is not difficult to verify that (3.1) implies that

$$
F=0, \quad \partial^{2} \phi=0, \quad p_{\mu} \gamma^{\mu} \psi=0 .
$$

It follows that the superfield $\Phi$ subject to the equation of motion (3.1) actually describes a free massless scalar and fermion.

In the case of $\mathcal{N}=1$ supersymmetry it is, of course, not difficult to find a manifestly supersymmetric offshell description of the theory. The equation of motion (3.1) follows by extremizing the action

$$
S=\int d^{2} \theta d^{3} x D_{\alpha} \bar{\Phi} D^{\alpha} \Phi
$$

w.r.t. $\Phi$. One way of adding interactions to the system (3.3) is to add a 'superpotential' term $\left(\int d^{2} \theta W(\Phi)\right)$ to the action; however we will not investigate offshell superspace in this paper.

\section{$3.2 \mathcal{N}=2$}

In this case the fermionic coordinates of superspace consist of two copies of the minimal $\mathcal{N}=1$ Majorana spinor which can be labeled as $\theta_{\alpha}^{i}(i=1,2)$. It is sometimes useful to group these coordinates into the complex pairs

$$
\theta_{\alpha}=\frac{1}{\sqrt{2}}\left(\theta_{\alpha}^{1}+i \theta_{\alpha}^{2}\right), \quad \bar{\theta}_{\alpha}=\frac{1}{\sqrt{2}}\left(\theta_{\alpha}^{1}-i \theta_{\alpha}^{2}\right) .
$$

In a similar manner there are two natural choices for a basis in the space of supersymmetries. One natural choice is to work with the supersymmetry operators defined in (2.1). The commutation relations of the supersymmetries (and associated supersymmetric derivatives) is given by

$$
\begin{aligned}
& \left\{Q_{\alpha}^{i}, Q_{\beta}^{j}\right\}=P_{\alpha \beta} \delta^{i j} \\
& \left\{D_{\alpha}^{i}, D_{\beta}^{j}\right\}=-P_{\alpha \beta} \delta^{i j}
\end{aligned}
$$


Another choice is to work with complex supersymmetries; if we define

$$
Q_{\alpha}=\frac{1}{\sqrt{2}}\left(Q_{\alpha}^{1}-i Q_{\alpha}^{2}\right), \quad D_{\alpha}=\frac{1}{\sqrt{2}}\left(D_{\alpha}^{1}-i D_{\alpha}^{2}\right)
$$

we have

$$
\begin{aligned}
& \left\{Q_{\alpha}, \bar{Q}_{\beta}\right\}=P_{\alpha \beta} \\
& \left\{D_{\alpha}, \bar{D}_{\beta}\right\}=-P_{\alpha \beta}
\end{aligned}
$$

(also $\left\{Q_{\alpha}, Q_{\beta}\right\}=\left\{D_{\alpha}, D_{\beta}\right\}=0$ ). In this basis the supersymmetry operators and supercovariant derivatives are most naturally written in terms of the complex variables $\theta_{\alpha}$; in particular for supercovariant derivatives we have

$$
\begin{aligned}
D_{\alpha} & =\frac{\partial}{\partial \theta^{\alpha}}+\frac{i}{2} \bar{\theta}^{\beta} \partial_{\beta \alpha} \\
\bar{D}_{\alpha} & =\frac{\partial}{\partial \bar{\theta}^{\alpha}}+\frac{i}{2} \theta^{\beta} \partial_{\beta \alpha}
\end{aligned}
$$

It is sometimes useful to utilize 'chiral' and anti chiral coordinates $\left(y_{R}, \theta_{\alpha}\right),\left(y_{L}, \bar{\theta}_{\alpha}\right)$ where

$$
y_{R}^{\mu}=x^{\mu}-\frac{i}{2} \theta \gamma^{\mu} \bar{\theta}, \quad y_{L}^{\mu}=x^{\mu}+\frac{i}{2} \theta \gamma^{\mu} \bar{\theta}
$$

These coordinates are useful because

$$
\bar{D}_{\alpha} y_{R}=0, \quad D_{\alpha} y_{L}=0
$$

It is easily verified that

$$
\begin{aligned}
D_{\alpha} & =\frac{\partial}{\partial \theta^{\alpha}}+i \bar{\theta}^{\beta} \partial_{\beta \alpha}^{y_{R}} \\
\bar{D}_{\alpha} & =\frac{\partial}{\partial \bar{\theta}^{\alpha}}
\end{aligned}
$$

Analogous expressions may also be obtained if we choose $y_{L}, \theta, \bar{\theta}$ as our coordinates.

$\mathcal{N}=2$ theories posses a $\mathrm{U}(1) R$-symmetry under which we can assign charges to operators. We normalize this symmetry by assigning the charges 1 and -1 to $\theta$ and $\bar{\theta}$ respectively. It follows that the operators $D_{\alpha}$ and $\bar{D}_{\alpha}$ respectively have charges -1 and +1 under $R$-symmetry. Below we will sometimes use the notation $D \leftrightarrow D^{--}$and $\bar{D} \leftrightarrow D^{++}$, notation that emphasizes these charge assignments.

The minimal free $\mathcal{N}=2$ theory has the same field content as the minimal $\mathcal{N}=1$ theory, i.e., the propagating degrees of freedom are a single complex scalar and complex fermion. The manifestly supersymmetric form of these equations of motion is given as follows. The basic dynamical superfield $\Phi$ is required to be chiral

$$
\bar{D}_{\alpha} \Phi=D_{\alpha} \bar{\Phi}=0
$$

In addition it is required to obey the equations of motion (of a free theory):

$$
D^{\alpha} D_{\alpha} \Phi=\bar{D}^{\alpha} \bar{D}_{\alpha} \bar{\Phi}=0
$$


These equations are solved by

$$
\Phi=\phi\left(y_{R}\right)+\theta \psi\left(y_{R}\right)=\phi+\theta \psi-\frac{i}{2} \theta \gamma^{\mu} \bar{\theta} \partial_{\mu} \phi
$$

and its complex conjugate (an anti-chiral field) is

$$
\bar{\Phi}=\bar{\phi}\left(y_{L}\right)-\theta \psi^{*}\left(y_{L}\right)=\bar{\phi}-\bar{\theta} \psi^{*}+\frac{i}{2} \theta \gamma^{\mu} \bar{\theta} \partial_{\mu} \bar{\phi}
$$

where $\phi$ and $\psi$ obey the free Klein Gordon and Dirac equations respectively (here $\theta \gamma^{\mu} \bar{\theta}=$ $\left.\theta^{\alpha}\left(\gamma^{\mu}\right)_{\alpha}^{\beta} \bar{\theta}_{\beta}\right)$.

As the field component of the minimal $\mathcal{N}=2$ theory is the same as that of the $\mathcal{N}=1$ theory, it is possible to write the $\mathcal{N}=2$ superfield in terms of the $\mathcal{N}=1$ superfield; explicitly

$$
\begin{aligned}
& \Phi_{\mathcal{N}=2}=\Phi_{\mathcal{N}=1}+i \theta^{(2)} D^{(1)} \Phi_{\mathcal{N}=1} \\
& \bar{\Phi}_{\mathcal{N}=2}=\bar{\Phi}_{\mathcal{N}=1}-i \theta^{(2)} D^{(1)} \bar{\Phi}_{\mathcal{N}=1}
\end{aligned}
$$

(here $\theta^{(2)}$ is the second Majorana Grassmann co-ordinate - the coordinate that belongs to $\mathcal{N}=2$ but not to $\mathcal{N}=1$ superspace - and the $\Phi_{\mathcal{N}=1}$ field has the usual expansion in the $\theta^{(1)}$ Grassmann co-ordinate.

\section{$3.3 \mathcal{N}=3$}

The fermionic coordinates of superspace consist of three Majorana spinors, $\theta_{\alpha}^{a}$ in this case. The indices $a$ transform in the vector representation of the $\mathrm{SO}(3) R$-symmetry. It is sometimes useful to regard vectors of the $\mathrm{SO}(3) R$-symmetry as bispinors, or $2 \times 2$ matrices. Vectors are easily converted to matrices by dotting their components with the Pauli matrices $\left(\sigma^{a}\right)_{i}{ }^{j}$.

The field content of the minimal $\mathcal{N}=3$ free theory consists of two free complex scalars and two free complex fermions. These fields may be packaged together into a doublet of complex superfields that transform in the spin- $\frac{1}{2}$ of the $R$-symmetry group. The free theory is a trivial example of a superconformal field theory. Primary operators in any superconformal field theory are labeled by $(\Delta, j, h)$ where $\Delta$ is the scaling dimension, $j$ is the spin and $h$ is the ' $R$-symmetry spin' (i.e. the quantum number that describes the $R$-symmetry representation of the primary operator). In this notation the free superfield described above transforms in the representation $\left(\frac{1}{2}, 0, \frac{1}{2}\right)$. The doublet of free superfields obey the 'equations of motion'

$$
D_{\alpha}^{\{i j} \Phi^{k\}}=0
$$

This equation of motion has a simple interpretation; it follows from the analysis of unitary representations of the superconformal algebra that a representation with quantum numbers $\left(\frac{1}{2}, 0, \frac{1}{2}\right)$ has a null state with quantum numbers $\left(1, \frac{1}{2}, \frac{3}{2}\right)$; the equation (3.13) is simply the assertion that this null state vanishes.

The equations of motion (3.13) may be analyzed as follows. Let us denote the two components of the doublet superfield $\Phi$ by $\Phi^{+}$and (superscripts denote $R$-symmetry charge; 
a single + denotes charge $\frac{1}{2}$ ). The equations of motion assert that

$$
\begin{aligned}
& 2 D_{\alpha}^{(3)} \Phi^{+}-\sqrt{2} D_{\alpha}^{++} \Phi^{-}=0 \\
& 2 D_{\alpha}^{(3)} \Phi^{-}+\sqrt{2} D_{\alpha}^{--} \Phi^{+}=0
\end{aligned}
$$

It is possible to solve for $\Phi^{+}$and $\Phi^{-}$in terms of a single $\mathcal{N}=2$ chiral superfield $\varphi^{+}$and a single antichiral superfield $\varphi^{-}$; we find

$$
\begin{aligned}
& \Phi^{+}=\varphi^{+}+\frac{1}{\sqrt{2}} \theta^{(3)} D^{++} \varphi^{-} \\
& \Phi^{-}=\varphi^{-}-\frac{1}{\sqrt{2}} \theta^{(3)} D^{--} \varphi^{+}
\end{aligned}
$$

These $\mathcal{N}=2$ superfields in turn obey the free $\mathcal{N}=2$ equations of motion

$$
D^{\alpha} D_{\alpha} \varphi^{+}=\bar{D}^{\alpha} \bar{D}_{\alpha} \varphi^{-}=0
$$

demonstrating that the propagating degrees of freedom are twice that of the $\mathcal{N}=2$ theory.

The final expression of the $\mathcal{N}=3$ superfield in terms of the component fields, after we have solved for the (3.16), is given by

$$
\Phi^{k}=\phi^{k}-\frac{1}{\sqrt{2}} \theta^{k l \alpha} \psi_{l \alpha}-\frac{1}{4} \epsilon^{a b c} \theta^{a \alpha} \theta^{b \beta}\left(\sigma^{c}\right)^{k l} \partial_{\alpha \beta} \phi_{l}+\frac{1}{12 \sqrt{2}} \epsilon^{a b c} \theta^{a \alpha} \theta^{b \beta} \theta^{c \gamma} \partial_{\alpha \beta} \psi_{\gamma}^{k}
$$

In the last term the $\alpha, \beta, \gamma$ indices are completely symmetrized and $k=1,2$. Here $a, b$ are vector $\mathrm{SO}(3)$ indices and $i, j, k$ are spinor indices. Note that (3.17) hold only when the component fields obey the free equations of motion.

\section{$3.4 \mathcal{N}=4$}

In this case we have four Majorana spinor coordinates $\theta_{\alpha}^{a}$ lying in the 4 of the $R$-symmetry group $\mathrm{SO}(4)$. The superfield $\Phi^{i}$ is a Weyl spinor of $\mathrm{SO}(4) .{ }^{4}$ The $\mathcal{N}=4$ chirality constraint is

$$
D_{\alpha}^{\tilde{i}\{j} \Phi^{k\}}=D_{\alpha}^{\tilde{i} j} \Phi^{k}+D_{\alpha}^{\tilde{i} k} \Phi^{j}=0 .
$$

To understand the field content of the minimal $\mathcal{N}=4$ theory, we split the $\mathcal{N}=4$ chirality constraint into a part that constrains the $\theta_{\alpha}^{(4)}$ dependence and a part that's purely $\mathcal{N}=3$. We begin by choosing an $\mathcal{N}=3$ subspace, which we take as the $1,2,3$ directions. The remaining 4 direction is the orthogonal direction. A chiral (top-half) part of a $\mathrm{SO}(4)$ Weyl spinor is the Dirac spinor in three dimensions. The $\mathrm{SO}(4)$ vector $D_{\alpha}^{(a)}$ decomposes into an $\mathrm{SO}(3)$ vector $D^{(a)}$ for $a=1,2,3$ and a scalar $D_{\alpha}^{(4)}$. This can be seen as the symmetric and antisymmetric part of the matrix $D_{\alpha}^{i \tilde{i}}$ respectively. The antisymmetric part contains only $D_{\alpha}^{(4)}$ and the symmetric part is the $D_{\alpha}^{i j}$ which is purely along the 1,2 and 3 directions. When the above chirality constraint is analyzed, one finds

$$
D_{\alpha}^{(4)} \Phi^{k}=-\frac{i}{3} D_{\alpha}^{k i} \Phi_{i}
$$

\footnotetext{
${ }^{4}$ See appendix A.2.2 for $\mathrm{SO}(4)$ conventions.
} 
where on the 1.h.s. we have the the supercovariant derivative along the 4 direction in the $\mathrm{SO}$ (4) $R$-symmetry space; on the r.h.s. we have the symmetric part of the $D_{\alpha}^{i \tilde{i}}$ supercovariant derivative, which is purely along the (orthogonal) $\mathrm{SO}(3)$ subspace. All the spinor indices are now thought of as $\mathrm{SO}(3)$ (Dirac) $\mathcal{N}=3$ spinor indices. This equation is the analog of (3.14) in the present case.

Solving (3.19) shows that the chiral $\mathcal{N}=4$ superfield $\Phi^{k}$ is completely determined in terms of a single $\mathcal{N}=3$ chiral superfield $\varphi_{i}$ as

$$
\Phi^{k}=\varphi^{k}-\frac{i}{3} \theta^{(4)} D^{k i} \varphi_{i}
$$

Thus, we see that the minimal field content of the $\mathcal{N}=4$ theory is the same as that of $\mathcal{N}=3$. An explicit component field expression can now be obtained from (3.20) by using (3.17) for the $\varphi^{k}$.

\section{$3.5 \mathcal{N}=6$}

In this case we have six Majorana spinor coordinates $\theta_{\alpha}^{a}$ lying in the vector representation of the $R$-symmetry group $\mathrm{SO}(6)$ (三 $\mathrm{SU}(4)$ ). The superfield $\Phi^{I}$ is a Weyl spinor of $\mathrm{SO}(6),{ }^{5}$ which is the 4 of $\mathrm{SU}(4)$. The field $\Phi^{I}$ satisfies the 'chirality constraint" ${ }^{6}$

$$
D_{\alpha}^{I J} \Phi^{K}=D_{\alpha}^{J K} \Phi^{I}=D_{\alpha}^{K I} \Phi^{J}
$$

To understand the field content of the minimal $\mathcal{N}=6$ theory, we proceed as above and split the $\mathcal{N}=6$ chirality constraint into an $\mathcal{N}=4$ part and another piece which describes the $\theta^{(5)}$ and $\theta^{(6)}$ dependences. We begin by choosing an $\mathcal{N}=4$ subspace, which we take as the 1,2, 3 and 4 directions. The remaining 5, 6 directions are the orthogonal directions. In the conventions we have chosen, it may be checked that a Weyl spinor $\Phi^{I}$ of $\mathrm{SO}(6)$ decomposes as one chiral $\Phi^{i}$ spinor $(i=1,2)$ and one anti-chiral (bottom half of the $\mathrm{SO}(4)$ spinor) $\Phi^{\tilde{i}}(\tilde{i}=3,4)$ of the $\mathrm{SO}(4)$ sub-group. ${ }^{7}$ Similarly, the $\mathrm{SO}(6)$ vector (the $(4 \times 4)$ antisym of $\mathrm{SU}(4))$ decomposes into two scalars and one $\mathrm{SO}(4)$ vector. In matrix language, one can construct the antisymmetric matrix $D_{\alpha}^{I J}$ and observe that the two scalars $\left(D_{\alpha}^{(5)}\right.$ and $\left.D_{\alpha}^{(6)}\right)$ form the linear combinations $i \sqrt{2} D_{\alpha}=D_{\alpha}^{(5)}-i D_{\alpha}^{(6)}$ and $i \sqrt{2} \bar{D}_{\alpha}=D_{\alpha}^{(5)}+i D_{\alpha}^{(6)}$, when $I, J=\tilde{i}, \tilde{j}$ and $I, J=i, j$ respectively. On the other hand, when $I, J=\tilde{i}, j$ (or vice-verse) we get the (single) vector which involves only $D_{\alpha}^{(a)}$ where $a=1, \ldots 4$. We can pick any two terms from the above equation (we choose the first and third) and analyze them as follows

$$
\begin{array}{ll}
D_{\alpha}^{i j} \Phi^{k}=D_{\alpha}^{k i} \Phi^{j} & D_{\alpha}^{\tilde{i j}} \Phi^{\tilde{k}}=D_{\alpha}^{\tilde{k} \tilde{i}} \Phi^{\tilde{j}} \\
D_{\alpha}^{\tilde{i} j} \Phi^{k}=D_{\alpha}^{k \tilde{i}} \Phi^{j} & D_{\alpha}^{i \tilde{j}} \Phi^{\tilde{k}}=D_{\alpha}^{\tilde{k} i} \Phi^{\tilde{j}} \\
D_{\alpha}^{i j} \Phi^{\tilde{k}}=D_{\alpha}^{\tilde{k} i} \Phi^{j} & D_{\alpha}^{\tilde{i j}} \Phi^{k}=D_{\alpha}^{k \tilde{i}} \Phi^{\tilde{j}}
\end{array}
$$

\footnotetext{
${ }^{5}$ See appendix A.2.3 for $\mathrm{SO}(6)$ conventions.

${ }^{6}$ We briefly use upper case $I, J$ which take values $1, \ldots 4$ for the SU(4) indices in (3.21) to avoid confusion with the lower case $i, j$ which appear in the $\mathcal{N}=4$ equations.

${ }^{7}$ We adopt the convention wherein the un-tilded indices $i$ take values 1,2 and the tilded indices $\tilde{i}$ take values 3,4 .
} 
The second equation in each of the two sets above is just the $\mathcal{N}=4$ chirality condition (3.18) for each of the fields $\Phi^{i}$ and $\Phi^{\tilde{i}}$. It remains to analyze the first and third equations from each of the two sets. The first set reads

$$
\bar{D}_{\alpha} \Phi^{k}=0 \quad D_{\alpha} \Phi^{\tilde{k}}=0
$$

where $\bar{D}_{\alpha}=\frac{1}{\sqrt{2}}\left(D_{\alpha}^{(5)}+i D_{\alpha}^{(6)}\right)$ and $D_{\alpha}=\frac{1}{\sqrt{2}}\left(D_{\alpha}^{(5)}-i D_{\alpha}^{(6)}\right)$. Thus, $\Phi^{k}$ and $\Phi^{\tilde{k}}$ can be thought of as two independent 'chiral' and 'anti-chiral' superfields and we can accordingly expand them in the $\theta_{\alpha}=\frac{1}{\sqrt{2}}\left(\theta_{\alpha}^{(5)}+i \theta_{\alpha}^{(6)}\right)$ and $\bar{\theta}_{\alpha}=\frac{1}{\sqrt{2}}\left(\theta_{\alpha}^{(5)}-i \theta_{\alpha}^{(6)}\right)$ coordinates. Let's now analyze the third equation from the above set. They are

$$
\bar{D}_{\alpha} \Phi^{\tilde{k}}=\frac{i}{2 \sqrt{2}} D_{\alpha}^{\tilde{k} i} \Phi_{i} \quad D_{\alpha} \Phi^{k}=\frac{i}{2 \sqrt{2}} D_{\alpha}^{k \tilde{i}} \Phi_{\tilde{i}}
$$

Solving the above equations leads us to the following result for $\Phi^{k}$ and $\Phi^{\tilde{k}}$

$$
\begin{aligned}
\Phi^{k} & =\varphi^{k}+\frac{i}{2 \sqrt{2}} \theta D^{k i} \varphi_{\tilde{i}}-\frac{i}{2} \theta \gamma^{\mu} \bar{\theta} \partial_{\mu} \varphi^{k} \\
\Phi^{\tilde{k}} & =\varphi^{\tilde{k}}+\frac{i}{2 \sqrt{2}} \bar{\theta} D^{\tilde{k} i} \varphi_{i}+\frac{i}{2} \theta \sigma^{\mu} \bar{\theta} \partial_{\mu} \varphi^{\tilde{k}}
\end{aligned}
$$

Where $k=1,2$ and $\tilde{k}=3,4$ make up the full $\mathcal{N}=6$ multiplet, and $\theta_{\alpha}=\frac{1}{\sqrt{2}}\left(\theta_{\alpha}^{(5)}+i \theta_{\alpha}^{(6)}\right)$ and $\bar{\theta}_{\alpha}=\frac{1}{\sqrt{2}}\left(\theta_{\alpha}^{(5)}-i \theta_{\alpha}^{(6)}\right)$. Thus, we see that the field content of the minimal $\mathcal{N}=6$ theory consists of two independent $\mathcal{N}=4$ fields, $\varphi^{k}$ and $\varphi^{\tilde{k}}$. (3.17) and (3.16) can now be used in (3.25) to obtain explicit component field expression for $\Phi^{K}$.

\section{Currents}

In this section we describe the construction of conserved currents in the theories discussed above. These currents constitute the full local gauge invariant operator spectrum of the theories considered. In the non-supersymmetric case the bosonic conserved currents and the violation, due to interactions, of their conservation by $\frac{1}{N}$ effects play a central role in the solution of three point functions in these theories $[3,4]$. The currents we consider in this section are the supersymmetric extension of the bosonic currents considered in $[3,4,17]$. We construct the supercurrents, using the onshell superspace described in sections 2 and 3 , in terms of onshell superfields and supercovariant derivatives.

\subsection{General structure of the current superfield}

Let us start by first describing the structure of the $\mathcal{N}=1$ supercurrents. A general spin $s$ supercurrent multiplet can be written as a superfield carrying $2 s$ spacetime spinor indices and can be expanded in components as follows

$$
\Phi^{\alpha_{1} \alpha_{2} \ldots \alpha_{2 s}}=\phi^{\alpha_{1} \alpha_{2} \ldots \alpha_{2 s}}+\theta_{\alpha} \psi^{\alpha \alpha_{1} \alpha_{2} \ldots \alpha_{2 s}}+\theta^{\left\{\alpha_{1}\right.} \chi^{\left.\alpha_{2} \ldots \alpha_{2 s}\right\}}+\theta^{\alpha} \theta_{\alpha} B^{\alpha_{1} \alpha_{2} \ldots \alpha_{2 s}}
$$

where all the indices $\alpha_{1}, \alpha_{2}, \ldots \alpha_{2 s}$ are symmetrized. The conservation (shortening) condition for the supercurrent is

$$
D_{\alpha_{1}} \Phi^{\alpha_{1} \alpha_{2} \ldots \alpha_{2 s}}=0
$$


where $D_{\alpha}$ is the supercovariant derivative given by

$$
D_{\alpha}=\frac{\partial}{\partial \theta^{\alpha}}+\frac{i}{2} \theta^{\beta} \partial_{\beta \alpha}
$$

Using eqs. (4.3) and (4.1) we obtain

$$
\begin{aligned}
\delta_{\alpha_{1}}^{\left\{\alpha_{1}\right.} \chi^{\left.\alpha_{2} \ldots \alpha_{2 s}\right\}} & +\theta_{\alpha_{1}}\left(2 B^{\alpha_{1} \alpha_{2} \ldots \alpha_{2 s}}-\frac{i}{2} \partial_{\beta}^{\alpha_{1}} \phi^{\beta \alpha_{2} \ldots \alpha_{2 s}}\right) \\
& -\frac{i}{2} \theta^{2} \partial_{\alpha \alpha_{1}} \psi^{\alpha \alpha_{1} \alpha_{2} \ldots \alpha_{2 s}}+\frac{i}{2} \theta^{\beta} \partial_{\beta \alpha_{1}} \theta^{\left\{\alpha_{1}\right.} \chi^{\left.\alpha_{2} \ldots \alpha_{2 s}\right\}}=0
\end{aligned}
$$

This implies

$$
\chi^{\alpha_{2} \ldots \alpha_{2 s}}=0
$$

while the symmetric part of the $\theta$ component gives

$$
B^{\alpha_{1} \alpha_{2} \ldots \alpha_{2 s}}=\frac{i}{4} \partial_{\beta}{ }^{\left\{\alpha_{1}\right.} \phi^{\left.|\beta| \alpha_{2} \ldots \alpha_{2 s}\right\}}
$$

whereas the antisymmetric part gives

$$
\epsilon_{\alpha_{1} \alpha_{2}} \partial_{\beta}^{\alpha_{1}} \phi^{\beta \alpha_{2} \ldots \alpha_{2 s}}=0 \Rightarrow \partial_{\alpha_{1} \alpha_{2}} \phi^{\alpha_{1} \alpha_{2} \ldots \alpha_{2 s}}=0
$$

which is the current conservation equation for the current $\phi$. Since $\chi=0$, the $\theta \theta$ component gives the current conservation equation for $\psi$

$$
\partial_{\alpha \alpha_{1}} \psi^{\alpha \alpha_{1} \ldots \alpha_{2 s}}=0
$$

Thus the form of the supercurrent multiplet for a spin $s$ conserved current is

$$
\Phi^{\alpha_{1} \alpha_{2} \ldots \alpha_{2 s}}=\phi^{\alpha_{1} \alpha_{2} \ldots \alpha_{2 s}}+\theta_{\alpha} \psi^{\alpha \alpha_{1} \alpha_{2} \ldots \alpha_{2 s}}+\frac{i}{4} \theta^{\alpha} \theta_{\alpha} \partial_{\beta}^{\left\{\alpha_{1}\right.} \phi^{\left.|\beta| \alpha_{2} \ldots \alpha_{2 s}\right\}}
$$

The general structure of the current superfield described above goes through for higher supersymmetries as well. For higher supersymmetries the conservation equation reads

$$
D_{\alpha_{1}}^{a} \Phi^{\alpha_{1} \alpha_{2} \ldots \alpha_{2 s}}=0
$$

where $a=1,2 \ldots \mathcal{N}$ is the R-symmetry index. ${ }^{8}$ In the case of an $\mathcal{N}=m$ spin- $s$ current multiplet, the currents $\phi^{\alpha_{1} \alpha_{2} \ldots \alpha_{2 s}}$ and $\psi^{\alpha \alpha_{1} \alpha_{2} \ldots \alpha_{2 s}}$ are themselves $\mathcal{N}=m-1$ spin $s$ and spin $s+\frac{1}{2}$ conserved current superfields (depending on the grassmann coordinates $\theta_{\alpha}^{a}$ : $a=1, \ldots m-1)$ while the $\theta_{\alpha}$ in $(4.9)$ is the left over grassmann coordinate $\theta_{\alpha}^{m}$. Thus we see the general structure of the supercurrent multiplets: an $\mathcal{N}=m$ spin $s$ supercurrent multiplet breaks up into two $\mathcal{N}=m-1$ supercurrents with spins $s$ and $s+\frac{1}{2}$ respectively.

\footnotetext{
${ }^{8}$ Note that for $\mathcal{N}>1,(4.10)$ is true only for R-symmetry singlet currents. For currents carrying nontrivial R-symmetry representation the shortening condition is different. In this paper we will only need the shortening condition (4.10).
} 
This structure can be used to express higher supercurrents superfields in term of components. For instance, the $\mathcal{N}=2$ spin $s$ currents superfield can be expanded in components as follows

$$
\begin{gathered}
\Phi^{\alpha_{1} \alpha_{2} \ldots \alpha_{2 s}}=\varphi^{\alpha_{1} \alpha_{2} \ldots \alpha_{2 s}}+\theta_{\alpha}^{a}\left(\psi^{a}\right)^{\alpha \alpha_{1} \alpha_{2} \ldots \alpha_{2 s}}+\frac{1}{2} \epsilon_{a b} \theta_{\alpha}^{a} \theta_{\beta}^{b} \mathcal{A}^{\alpha \beta \alpha_{1} \alpha_{2} \ldots \alpha_{2 s}} \\
+ \text { term involving derivatives of } \varphi, \psi^{a} \text { and } \mathcal{A}
\end{gathered}
$$

where $a, b$ are R-symmetry indices and take values in $\{1,2\}$. The conformal state content so obtained, namely $\left(\varphi, \psi^{1}, \psi^{2}, \mathcal{A}\right)$ above, match exactly with the decomposition of spin $s$ supercurrent multiplet into conformal multiplets presented in appendix C.2.

\subsection{Free field construction of currents}

In this section we give explicit construction of the conserved supercurrent discussed in previous subsection in term of free superfields.

\subsection{1 $\mathcal{N}=1$}

The spin $s$ supercurrent here can be expressed in term of the $\mathcal{N}=1$ superfield $\Phi$ as follows

$$
J^{(s)}=\sum_{r=0}^{2 s}(-1)^{\frac{r(r+1)}{2}}\left(\begin{array}{c}
2 s \\
r
\end{array}\right) D^{r} \bar{\Phi} D^{2 s-r} \Phi
$$

where $J^{(s)}=\lambda^{\alpha_{1}} \lambda^{\alpha_{2}} \cdots \lambda^{\alpha_{2 s}} J_{\alpha_{1} \alpha_{2} \cdots \alpha_{2 s}}$ and $D=\lambda^{\alpha} D_{\alpha}$, and $\lambda_{\alpha}$ s are polarization spinors and $s=0, \frac{1}{2}, 1 \ldots$ The currents are of both integral and half-integral spins. It can be verified that the above is the unique expression for the conserved spin- $s$ current in $\mathcal{N}=1$ free field theory. In equations, the following holds

$$
\frac{\partial}{\partial \lambda^{\alpha}} D^{\alpha} J^{(s)}=0 .
$$

We note here that the stress tensor lies in the spin $\frac{3}{2}$ current supermultiplet (which also contains the supersymmetry current), and thus is conserved exactly even in interacting theory.

\subsection{2 $\mathcal{N}=2$}

We give the expression of the conserved current in terms of the free $\mathcal{N}=2$ superfield $\Phi$ and its complex conjugate $\bar{\Phi}$.

$$
J^{(s)}=\sum_{r=0}^{s}\left\{(-1)^{r(2 r+1)}\left(\begin{array}{c}
2 s \\
2 r
\end{array}\right) \partial^{r} \bar{\Phi} \partial^{s-r} \Phi+(-1)^{(r+1)(2 r+1)}\left(\begin{array}{c}
2 s \\
2 r+1
\end{array}\right) \partial^{r} \bar{D} \bar{\Phi} \partial^{s-r-1} D \Phi\right\}
$$

where $\partial=i \lambda^{\alpha} \gamma_{\alpha \beta}^{\mu} \lambda^{\beta} \partial_{\mu}, D=\lambda^{\alpha} D_{\alpha}$ and $s=0,1,2 \ldots$ The spin 1 supercurrent multiplet contains the stress tensor, supersymmetry current and $R$-current, and its conservation holds even in the interacting superconformal theory.

As described above in subsection 4.1 these $\mathcal{N}=2$ currents can be decomposed into $\mathcal{N}=1$ currents. It is straightforward to check that the currents (4.14) when expanded in $\theta_{\alpha}^{2}$ as in (4.9) correctly reproduce the $\mathcal{N}=1$ currents (4.12). This give a consistency check of these $\mathcal{N}=2$ currents. 


\subsection{3 $\mathcal{N}=3$}

The $\mathcal{N}=3$ chirality constraint on the matter superfield $\Phi^{k}$ is

$$
\begin{aligned}
D^{\{i j} \Phi^{k\}} & =D^{i j} \Phi^{k}+D^{i k} \Phi^{j}+D^{j k} \Phi^{i}=0 \\
\text { or equivalently } \quad D^{i j} \Phi^{k} & =-\frac{1}{3}\left(D^{i l} \Phi_{l} \epsilon^{j k}+D^{j l} \Phi_{l} \epsilon^{i k}\right)
\end{aligned}
$$

where $D_{\alpha}^{i j}=\left(\sigma^{a}\right)^{i j} D_{\alpha}^{a}$.

From this chirality constraint the following identities, which would be useful in proving current conservation, can be derived ${ }^{9}$

$$
D_{\alpha}^{i j} D_{\beta}^{m n} \Phi^{k}=\frac{1}{2}\left(i \partial_{\alpha \beta} \Phi^{i} \epsilon^{j m} \epsilon^{n k}+i \partial_{\alpha \beta} \Phi^{i} \epsilon^{j n} \epsilon^{m k}+i \partial_{\alpha \beta} \Phi^{j} \epsilon^{i m} \epsilon^{n k}+i \partial_{\alpha \beta} \Phi^{j} \epsilon^{\mathrm{in}} \epsilon^{m k}\right)
$$

Contracting various indices, the following relations can be obtained from (4.16) as corollaries

$$
\begin{aligned}
D^{\alpha i j} D_{\alpha}^{m n} \Phi^{k} & =0 \\
D_{\alpha}^{i j} D_{\beta}^{m k} \Phi_{k} & =-\frac{3}{2}\left(i \partial_{\alpha \beta} \Phi^{i} \epsilon^{j m}+i \partial_{\alpha \beta} \Phi^{j} \epsilon^{i m}\right) \\
D_{\alpha}^{i j} D_{i j \beta} \Phi^{k} & =-3 i \partial_{\alpha \beta} \Phi^{k}=\frac{2}{3} D_{j}^{k} D^{j i} \Phi_{i}
\end{aligned}
$$

We give here the expression for the conserved currents in terms of the $\mathcal{N}=3$ superfield $\Phi^{i}$.

$$
\begin{aligned}
J^{(s)} & =\sum_{r=0}^{s}(-1)^{r}\left(\begin{array}{c}
2 s \\
2 r
\end{array}\right) \partial^{r} \bar{\Phi}^{i} \partial^{s-r} \Phi_{i}+\frac{2}{9} \sum_{r=0}^{s-1}(-1)^{r+1}\left(\begin{array}{c}
2 s \\
2 r+1
\end{array}\right) \partial^{r} D_{i}{ }^{j} \bar{\Phi}^{i} \partial^{s-r-1} D_{j}{ }^{k} \Phi_{k} \\
J^{\left(s+\frac{1}{2}\right)} & =\sum_{r=0}^{s}\left\{(-1)^{r}\left(\begin{array}{c}
2 s+1 \\
2 r
\end{array}\right) \partial^{r} \bar{\Phi}^{i} \partial^{s-r} D_{i}^{j} \Phi_{j}+(-1)^{r+1}\left(\begin{array}{c}
2 s+1 \\
2 r+1
\end{array}\right) \partial^{r} D_{i}^{j} \bar{\Phi}^{i} \partial^{s-r} \Phi_{j}\right\}
\end{aligned}
$$

where $\partial=i \lambda^{\alpha} \gamma_{\alpha \beta}^{\mu} \lambda^{\beta} \partial_{\mu}, D=\lambda^{\alpha} D_{\alpha}$ and $s=0,1,2 \ldots$ The stress energy tensor in this case lies the spin $\frac{1}{2}$ supercurrent multiplet along with the $R$-current and supersymmetry currents. The conservation of this supercurrent holds exactly even in the interacting superconformal theory.

\subsection{4 $\mathcal{N}=4$}

The $R$-symmetry in this case is $\mathrm{SO}(4)$ (equivalently $\left.\mathrm{SU}(2)_{l} \times \mathrm{SU}(2)_{r}\right) .{ }^{10}$ The supercharges $Q_{\alpha}^{\tilde{i} i}$ transform in the 4 of $\mathrm{SO}(4)$ (equivalently $(2,2)$ of $\left.\mathrm{SU}(2)_{l} \times \mathrm{SU}(2)_{r}\right)$. The two matter superfields transform in the $(2,0)$ representation which implies that the scalar transforms in the $(2,0)$ while the fermions transform in $(0,2)$. The matter multiplet again satisfies a 'chirality' constraint

$$
\begin{aligned}
D^{\tilde{i}\{i} \Phi^{j\}} & =D^{\tilde{i} i} \Phi^{j}+D^{\tilde{i} j} \Phi^{i}=0, \\
\text { or equivalently } \quad D^{\tilde{i} i} \Phi^{j} & =-\frac{1}{2} \epsilon^{i j} D_{\alpha}^{\tilde{i} k} \Phi_{k} .
\end{aligned}
$$

\footnotetext{
${ }^{9}$ See appendix A.2.1 for $\mathrm{SO}(3)$ conventions.

${ }^{10}$ The indices $a, b \ldots$ take values $1,2,3,4$ and represent the vector indices of $\mathrm{SO}(4)$ while the fundamental indices of the $\mathrm{SU}(2)_{l}$ and $\mathrm{SU}(2)_{r}$ are denoted by $i, j \ldots$ and $\tilde{i}, \tilde{j} \ldots$
} 
where $D_{\alpha}^{\tilde{i j}}=\left(\bar{\sigma}^{a}\right)^{\tilde{i} j} D_{\alpha}^{a}$.

From this chirality constraint the following identities, useful in proving current conservation, can be derived ${ }^{1}$

$$
D_{\alpha}^{\tilde{i} i} D_{\beta}^{\tilde{j} j} \Phi^{k}=2 i \partial_{\alpha \beta} \Phi^{i} \epsilon^{\tilde{i} \tilde{j}} \epsilon^{j k}
$$

Contracting various indices, the following equations can be obtained from (4.20) as corollaries

$$
\begin{aligned}
D^{\alpha \tilde{i} i} D_{\alpha}^{\tilde{j} j} \Phi^{k} & =0 \\
D_{\alpha}^{\tilde{i} i} D_{\beta}^{\tilde{j} j} \Phi_{j} & =-4 i \partial_{\alpha \beta} \Phi^{i} \epsilon^{\tilde{i} \tilde{j}} \\
D_{\alpha}^{\tilde{i} j} D_{\beta \tilde{i}} \Phi^{k} & =2 D_{\alpha}^{\tilde{i i}} D_{\beta \tilde{i i}} \Phi^{j}=8 i \partial_{\alpha \beta} \Phi^{j} .
\end{aligned}
$$

Using these equations it is straightforward to show that the following currents are conserved.

$$
J^{(s)}=\sum_{r=0}^{s}(-1)^{r}\left(\begin{array}{l}
2 s \\
2 r
\end{array}\right) \partial^{r} \bar{\Phi}^{i} \partial^{s-r} \Phi_{i}+\frac{1}{8} \sum_{r=0}^{s-1}(-1)^{r}\left(\begin{array}{c}
2 s \\
2 r+1
\end{array}\right) \partial^{r} D^{\tilde{i} i} \bar{\Phi}_{i} \partial^{s-r-1} D_{\tilde{i} j} \Phi^{j} .
$$

where $\partial=i \lambda^{\alpha} \gamma_{\alpha \beta}^{\mu} \lambda^{\beta} \partial_{\mu}, D=\lambda^{\alpha} D_{\alpha}$ and $s=0,1,2 \ldots$ In this theory the stress energy tensor lies in the $R$-symmetry singlet spin zero supercurrent multiplet $(1,0,\{0,0\})$.

\subsection{5 $\mathcal{N}=6$}

The field content of this theory is double of the field content of the $\mathcal{N}=4$ theory. In $\mathcal{N}=2$ language the field content is 2 chiral and 2 antichiral multiplets in fundamental of the gauge group. The $R$-symmetry in this theory is $\mathrm{SO}(6)$ ( $\equiv \mathrm{SU}(4)$ ) under which the supercharges transform in vector representation $(6$ of $\mathrm{SO}(6))$ while the $2+2$ chiral and antichiral multiplets transform in chiral spinor representation (4 of SU(4)).

The $\mathcal{N}=6$ shortening (chirality) condition on the matter multiplet is ${ }^{11}$

$$
\begin{aligned}
D_{\alpha}^{i j} \Phi^{k} & =D_{\alpha}^{j k} \Phi^{i}=D_{\alpha}^{k i} \Phi^{j} \\
\text { or equivalently } \quad D_{\alpha}^{a} \Phi^{k} & =-\frac{1}{10} D_{\alpha}^{b} \Phi^{l}\left(\bar{\gamma}^{a b}\right)_{l}{ }^{k}
\end{aligned}
$$

From this chirality constraint the following identities, which are useful in proving current conservation, can be derived ${ }^{2}$

$$
\begin{aligned}
D_{\alpha}^{a} D_{\beta}^{b} \Phi^{k} & =\frac{i}{2} \partial_{\alpha \beta} \Phi^{k} \delta^{a b}+\frac{i}{4} \partial_{\alpha \beta} \Phi^{l}\left(\bar{\gamma}^{a b}\right)_{l}{ }^{k}, \\
\text { or equivalently } \quad D_{\alpha}^{i j} D_{\beta}^{m n} \Phi^{k} & =-i \partial_{\alpha \beta}\left(\epsilon^{i j m n} \Phi^{k}+\epsilon^{k j m n} \Phi^{i}+\epsilon^{i k m n} \Phi^{j}-\epsilon^{i j k n} \Phi^{m}-\epsilon^{i j m k} \Phi^{n}\right)
\end{aligned}
$$

Taking the complex conjugate of equations (4.23) and (4.24), and using the property that $\gamma^{a b}$ and $\bar{\gamma}^{a b}$ are antihermitian, we get

$$
\begin{aligned}
D_{\alpha}^{i j} \bar{\Phi}_{k} & =\frac{1}{3}\left(D_{\alpha}^{i l} \bar{\Phi}_{l} \delta_{k}^{j}-D_{\alpha}^{j l} \bar{\Phi}_{l} \delta_{k}^{i}\right) \\
\text { or equivalently } \quad D_{\alpha}^{a} \bar{\Phi}_{k} & =\frac{1}{10} D_{\alpha}^{b}\left(\bar{\gamma}^{a b}\right)_{k}^{l} \bar{\Phi}_{l}
\end{aligned}
$$

\footnotetext{
${ }^{11}$ Here we revert back to lower case letters for the $\mathrm{SU}(4)$ indices $i, j$ (taking values $1, \ldots 4$ ) as there is no confusion with other $R$ indices.
} 
and

$$
\begin{aligned}
D_{\alpha}^{a} D_{\beta}^{b} \bar{\Phi}_{k} & =\frac{i}{2} \partial_{\alpha \beta} \bar{\Phi}_{k} \delta^{a b}-\frac{i}{4} \partial_{\alpha \beta}\left(\bar{\gamma}^{a b}\right)_{k}^{l} \bar{\Phi}_{l}, \\
\text { or equivalently } \quad D_{\alpha}^{i j} D_{\beta}^{m n} \bar{\Phi}_{k} & =-i \partial_{\alpha \beta}\left(\epsilon^{i j m n} \Phi^{k}-\epsilon^{l j m n} \bar{\Phi}_{l} \delta_{k}^{i}-\epsilon^{i l m n} \bar{\Phi}_{l} \delta_{k}^{j}+\epsilon^{i j l n} \bar{\Phi}_{l} \delta_{k}^{m}+\epsilon^{i j m l} \bar{\Phi}_{l} \delta_{k}^{n}\right)
\end{aligned}
$$

Using the above relation a straightforward computation shows that the following $R$ symmetry singlet integer spin currents are conserved

$J^{(s)}=\sum_{r=0}^{s}(-1)^{r}\left(\begin{array}{l}2 s \\ 2 r\end{array}\right) \partial^{r} \bar{\Phi}_{p} \partial^{s-r} \Phi^{p}-\frac{1}{24} \sum_{r=0}^{s-1}(-1)^{r+1}\left(\begin{array}{c}2 s \\ 2 r+1\end{array}\right) \epsilon_{i j k l} \partial^{r} D^{i j} \bar{\Phi}_{p} \partial^{s-r-1} D^{k l} \Phi^{p}$.

where $\partial=i \lambda^{\alpha} \gamma_{\alpha \beta}^{\mu} \lambda^{\beta} \partial_{\mu}, D=\lambda^{\alpha} D_{\alpha}$ and $s=0,1,2 \ldots$ The stress-energy tensor of this theory lies, as in the $\mathcal{N}=4$ theory, in the R-symmetry singlet spin zero multiplet $(1,0,\{0,0,0\})$.

\section{Weakly broken conservation}

The free superconformal theories discussed above have an exact higher spin symmetry algebra generated by the charges corresponding to the infinite number of conserved currents that these theories possess. These free theories can be deformed into interacting theories by turning on $\mathrm{U}(N)(\mathrm{SU}(N))$ Chern-Simons(CS) gauge interactions, in a supersymmetric fashion and preserving the conformal invariance of free CFTs, under which the matter fields transform in fundamental representations. The CS gauge interactions do not introduce any new local degrees of freedom so the spectrum of local operators in the theory remains unchanged. Turning on the interactions breaks the higher spin symmetry of the free theory but in a controlled way which we discuss below. These interacting CS vector models are interesting in there own right as non trivial interacting quantum field theories. Exploring the phase structure of these theories at finite temperature and chemical potential, provides a platform for studying a lot of interesting physics, at least in the large $N$ limit, using the techniques developed in [2].

From a more string theoretic point of view, a very interesting example of this class of theories is the $\mathrm{U}(N) \times \mathrm{U}(M)$ ABJ theory in the vector model limit $\frac{M}{N} \rightarrow 0$. ABJ theory in this vector model limit has recently been argued to be holographically dual a non-abelian supersymmetric generalization of the non-minimal Vasiliev theory in $A d S_{4}$ [6]. The ABJ theory thus connects, as its holographic duals, Vasiliev theory at one end to a string theory at another end. Increasing $\frac{M}{N}$ from 0 corresponds to increasing the coupling of $\mathrm{U}(M)$ gauge interactions in the bulk Vasiliev theory. Thus, understanding the ABJ theory away from the vector model limit in an expansions in $\frac{M}{N}$ would be a first step towards understanding of how string theory emerges from 'quantum' Vasiliev theory. ${ }^{12}$

In $[3,4]$ theories with exact conformal symmetry but weakly broken higher spin symmetry were studied. It was first observed in [2], and later used with great efficiency in [4],

\footnotetext{
${ }^{12}$ See [26] for a very recent attempt in this direction.
} 
that the anomalous "conservation" equations are of the schematic form

$$
\partial \cdot J_{(s)}=\frac{a}{N} J_{\left(s_{1}\right)} J_{\left(s_{2}\right)}+\frac{b}{N^{2}} J_{\left(s_{1}^{\prime}\right)} J_{\left(s_{2}^{\prime}\right)} J_{\left(s_{3}^{\prime}\right)}
$$

plus derivatives sprinkled appropriately. The structure of this equation is constrained on symmetry grounds - the twist $\left(\Delta_{i}-s_{i}\right)$ of the l.h.s. is 3 . If each $J_{s}$ has conformal dimension $\Delta=s+1+O(1 / N)$, and thus twist $\tau=1+O(1 / N)$, the two terms on the r.h.s. are the only ones possible by twist matching. Thus we can have only double or triple trace deformations in the case of weakly broken conservation and terms with four or higher number of currents are not possible.

In the superconformal case that we are dealing with, since $D$ has dimension $1 / 2$, $D \cdot J_{(s)}$ is a twist 2 operator. Thus in this case the triple trace deformation is forbidden and the only possible structure is more constrained:

$$
D \cdot J_{(s)}=\frac{a}{N} J_{\left(s_{1}\right)} J_{\left(s_{2}\right)}
$$

In view of this, it is feasible that in large-N supersymmetric Chern-Simons theories the structure of correlation functions is much more constrained (compared to the nonsupersymmetric case).

\section{Two-point functions}

The two-point function of two spin- $s$ operators in a 3d SCFT has a form completely determined (upto overall multiplicative constants) by superconformal invariance. Since, as we saw in section $2, X_{12 \pm}$ is the only superconformally covariant structure built out of two points in superspace, the only possible expression for the two point function which also has the right dimension and homogeneity in $\lambda$ is:

$$
\left\langle J_{s}(1) J_{s}(2)\right\rangle \propto \frac{P_{3}^{2 s}}{\tilde{X}_{12}^{2}}
$$

where $P_{3}$ is the superconformal invariant defined on two points, given in table 1 . The overall constant can be determined in free field theory, see below.

As an illustrative example, we consider the two-point function of two spin half supercurrents. On the basis of symmetry and dimension matching we can have the following possible structure for the 2-point function:

$$
\left\langle J_{1 / 2}\left(x_{1}, \theta_{1}, \lambda_{1}\right) J_{1 / 2}\left(x_{2}, \theta_{2}, \lambda_{2}\right)\right\rangle=b \frac{\lambda_{1} \lambda_{2}}{\tilde{X}_{12}^{\Delta_{1}+\Delta_{2}}} \frac{\theta_{12}^{2}}{\tilde{X}_{12}}+\frac{\lambda_{1} \tilde{X}_{12} \lambda_{2}}{\tilde{X}_{12}^{\Delta_{1}+\Delta_{2}+1}}\left(c+d \frac{\theta_{12}^{2}}{\tilde{X}_{12}}\right)
$$

where $\tilde{X}_{12} \equiv \sqrt{\left(\tilde{X}_{12}\right)_{\alpha}^{\beta}\left(\tilde{X}_{12}\right)_{\beta}^{\alpha}} \cdot{ }^{13}$ The shortening condition on the above 2-point function gives

$$
d=0 \quad b=\frac{i c}{4}\left(\Delta_{1}+\Delta_{2}-2\right)
$$

\footnotetext{
${ }^{13}$ Note that throughout $\tilde{X}_{12}$ denotes this scalar object. The matrix will always be denoted with the indices: $\left(\tilde{X}_{12}\right)_{\alpha}^{\beta}$.
} 
For $J_{1 / 2}$ a superconformal primary $\Delta_{1}=\Delta_{2}=3 / 2$ so $b=i c / 4$ and the two point function (upto some undetermined overall normalization) is given by

$$
\left\langle J_{1 / 2}\left(x_{1}, \theta_{1}, \lambda_{1}\right) J_{1 / 2}\left(x_{2}, \theta_{2}, \lambda_{2}\right)\right\rangle \propto \frac{\lambda_{1} \tilde{X}_{12} \lambda_{2}}{\tilde{X}_{12}^{4}}+\frac{i}{4} \frac{\lambda_{1} \lambda_{2} \theta_{12}^{2}}{\tilde{X}_{12}^{4}}
$$

A natural generalization, that reduces correctly to the above equation for $s=1 / 2$, is

$$
\left\langle J_{s}(1) J_{s}(2)\right\rangle \propto \frac{\left(\lambda_{1} \tilde{X}_{12} \lambda_{2}\right)^{2 s-1}}{\tilde{X}_{12}^{4 s+2}}\left(\lambda_{1} \tilde{X}_{12} \lambda_{2}+\frac{i s}{2} \lambda_{1} \lambda_{2} \theta_{12}^{2}\right)
$$

with $\left\langle J_{0} J_{0}\right\rangle=1 / \tilde{X}_{12}^{2}$ (since the superconformal shortening condition is different for spin zero). Note that the above can be written as

$$
\left\langle J_{s}(1) J_{s}(2)\right\rangle \propto \frac{\left(\lambda_{1} \tilde{X}_{12} \lambda_{2}+\frac{i}{4} \lambda_{1} \lambda_{2} \theta_{12}^{2}\right)^{2 s}}{\tilde{X}_{12}^{4 s+2}}
$$

which is the same as (6.1). The shortening condition on this is satisfied, as may be explicitly checked.

As a check, we also work out, by elementary field theory methods, the two point function of the spin $\frac{1}{2}$ current constructed out of the free $\mathcal{N}=1$ superfield which is defined as ${ }^{14}$

$$
\begin{aligned}
& \Phi=\phi+i \theta \psi \\
& \bar{\Phi}=\bar{\phi}+i \theta \psi^{*}
\end{aligned}
$$

We find that the 2-point function computed explicitly in the free theory is in agreement with our result (6.1) above. The spin half supercurrent is

$$
J_{\alpha}=\bar{\Phi} D_{\alpha} \Phi-\left(D_{\alpha} \bar{\Phi}\right) \Phi
$$

Using the equation of motion for $\Phi$ this obeys the shortening condition $D^{\alpha} J_{\alpha}=0$. The two point function of two such currents can be obtained after doing Wick contractions to write 4-point functions in terms of 2-point functions. We use the free field propagator $\langle\bar{\Phi} \Phi\rangle=\frac{1}{\tilde{X}_{12}}$, and also that

$$
D_{1 \alpha} D_{2 \beta} \frac{1}{\tilde{X}_{12}}=\frac{-i\left(\tilde{X}_{12}\right)_{\alpha \beta}}{\left(\tilde{X}_{12}\right)^{3}}, \quad D_{1 \alpha} \frac{1}{\tilde{X}_{12}} D_{2 \beta} \frac{1}{\tilde{X}_{12}}=\frac{\epsilon_{\alpha \beta} \theta_{12}^{2}}{4\left(\tilde{X}_{12}\right)^{4}}
$$

This gives (upto multiplicative factors which we neglect)

$$
\left\langle J_{\alpha}(1) J_{\beta}(2)\right\rangle \propto \frac{\left(\left(-\tilde{X}_{12}\right)_{\alpha \beta}+\frac{i}{4} \theta_{12}^{2} \epsilon_{\alpha \beta}\right)}{\tilde{X}_{12}^{4}} .
$$

Contracting with $\lambda_{1}^{\alpha}$ and $\lambda_{2}^{\beta}$ we find, in free field theory,

$$
\left\langle J_{\frac{1}{2}}(1) J_{\frac{1}{2}}(2)\right\rangle=-i \frac{P_{3}}{\tilde{X}_{12}^{2}}
$$

\footnotetext{
${ }^{14}$ We insert a factor of $i$ in this definition for convenience, which differs from the definition given in (3.2).
} 


\begin{tabular}{|c|c|c|}
\hline & Parity even & Parity odd \\
\hline Bosonic & $\begin{array}{c}P_{1}=\lambda_{2} X_{23-}^{-1} \lambda_{3} \\
Q_{1}=\lambda_{1} X_{12-}^{-1} X_{23+} X_{31-}^{-1} \lambda_{1} \\
\text { and cyclic }\end{array}$ & $\begin{array}{c}S_{1}=\frac{\lambda_{3} X_{31+} X_{12}+\lambda_{2}}{\tilde{X}_{12} \tilde{X}_{23} \tilde{X}_{31}} \\
\text { and cyclic }\end{array}$ \\
\hline Fermionic & $R_{1}=\lambda_{1} \Theta_{1}$ and cyclic & $T=\tilde{X}_{31} \frac{\Theta_{1} X_{12+} X_{23+} \Theta_{3}}{\tilde{X}_{12} \tilde{X}_{23}}$ \\
\hline
\end{tabular}

Table 1. Invariant structures in $\mathcal{N}=1$ superspace.

which, indeed, is what was expected. One can determine the constants appearing in front of the two point function in free field theory and we divide by it (so that the final result is normalized to one) which gives the following result for general spin $s$

$$
\left\langle J_{s}(1) J_{s}(2)\right\rangle=c(s) \frac{P_{3}^{2 s}}{\tilde{X}_{12}^{2}}
$$

where $c(s)=\left(\frac{i}{2}\right)^{2 s} \frac{\sqrt{\pi}}{s ! \Gamma\left(s+\frac{1}{2}\right)}$ for all $s \geq 0$.

\section{Three-point functions}

In this section we undertake the task of determining all the possible structures that can occur in the three-point functions of higher spin operators $\left\langle J_{s_{1}} J_{s_{2}} J_{s_{3}}\right\rangle$. For the nonsupersymmetric case this was done in [17]. We will use superconformal invariance to ascertain what structures can occur in three-point functions.

The structure of correlation functions in SCFTs has been earlier studied by $\mathrm{J}-\mathrm{H}$ Park [18, 20-22] and H. Osborn [19]. ${ }^{15}$ The structure of covariant objects which are used as building blocks for the construction of invariants in the present work was entirely laid out in the above references. However, our goal in the present work is to make use of these structures to study theories which have conserved currents of higher spin. For this purpose, it is convenient to adopt the polarization spinor formalism of [17]. After writing down the structures that can appear for a given three-point function, we use on-shell conservation laws of the currents to constrain the coefficients appearing in front of the structures.

We find that there exist new structures for both the parity even and odd part of $\left\langle J_{s_{1}} J_{s_{2}} J_{s_{3}}\right\rangle$ which were not present in the nonsupersymmetric case. The parity-odd superconformal invariants are of special interest as they arise in interacting $3 \mathrm{~d}$ SCFTs. We will here restrict ourselves to the case of $\mathcal{N}=1$ SCFTs (no $R$-symmetry). The results are summarized in the table given below:

\footnotetext{
${ }^{15}$ Kuzenko [27] has also studied 3-pt functions of the supercurrent and flavour currents of $\mathcal{N}=24 \mathrm{~d}$ SCFTs.
} 


\subsection{Superconformal invariants for three-point functions of $\mathcal{N}=1$ higher spin operators}

We need to determine all the superconformal invariants that can be constructed out of the co-ordinates of (augmented) superspace: $x_{i}, \theta_{i}$ and the (bosonic) polarization spinors $\lambda_{i}$ $(i=1,2,3)$. Using the covariant objects of section 2 , which transformed homogeneously under superinversions, we can begin to write down the superconformal invariants constructed out of $\left(x_{i}, \theta_{i}, \lambda_{i}\right)$.

We have

$$
\lambda_{i} X_{i j-}^{-1} \lambda_{j} \rightarrow-\left(\lambda_{i} X_{i-}^{-1}\right)\left(-X_{i-} X_{i j-}^{-1} X_{j-}\right)\left(X_{j+}^{-1} \lambda_{j}\right)=\lambda_{i} X_{i j-}^{-1} \lambda_{j}
$$

Thus we have the three superconformal invariants

$$
P_{1}=\lambda_{2} X_{23-}^{-1} \lambda_{3}, \quad P_{2}=\lambda_{3} X_{31-}^{-1} \lambda_{1}, \quad P_{3}=\lambda_{1} X_{12-}^{-1} \lambda_{2}
$$

Also, under superinversion,

$$
\mathfrak{X}_{1+}=X_{12-}^{-1} X_{23+} X_{31-}^{-1} \rightarrow-X_{1-} \mathfrak{X}_{1+} X_{1+}
$$

and similarly for $\mathfrak{X}_{2+}, \mathfrak{X}_{3+}$, so we also have the following as superconformal invariants:

$$
Q_{1}=\lambda_{1} \mathfrak{X}_{1+} \lambda_{1}, \quad Q_{2}=\lambda_{2} \mathfrak{X}_{2+} \lambda_{2}, \quad Q_{3}=\lambda_{3} \mathfrak{X}_{3+} \lambda_{3}
$$

Furthermore,

$$
\lambda_{3} X_{31+} X_{12+} \lambda_{2} \rightarrow-\frac{1}{x_{1}^{2} x_{2}^{2} x_{3}^{2}} \lambda_{3} X_{31+} X_{12+} \lambda_{2}, \quad \tilde{X}_{i j}^{2} \rightarrow \frac{\tilde{X}_{i j}^{2}}{x_{i}^{2} x_{j}^{2}}
$$

so there are the additional (parity odd) superconformal invariants

$$
S_{1}=\frac{\lambda_{3} X_{31+} X_{12+} \lambda_{2}}{\tilde{X}_{12} \tilde{X}_{23} \tilde{X}_{31}}, \quad S_{2}=\frac{\lambda_{1} X_{12+} X_{23+} \lambda_{3}}{\tilde{X}_{12} \tilde{X}_{23} \tilde{X}_{31}}, \quad S_{3}=\frac{\lambda_{2} X_{23+} X_{31+} \lambda_{1}}{\tilde{X}_{12} \tilde{X}_{23} \tilde{X}_{31}}
$$

which transform to minus themselves under inversion. Together these constitute the supersymmetric generalizations of the conformally invariant $P, Q, S$ structures discussed in $[17] .^{16}$

Using the covariant $\Theta$ structures of section 2 it follows that we have the additional (parity even) fermionic invariants

$$
R_{1}=\lambda_{1} \Theta_{1}, \quad R_{2}=\lambda_{2} \Theta_{2}, \quad R_{3}=\lambda_{3} \Theta_{3}
$$

It may be checked that

$$
R_{1}^{2}=R_{2}^{2}=R_{3}^{2}=R_{1} R_{2} R_{3}=0
$$

\subsubsection{Construction of the parity odd fermionic invariant $T$}

We can construct more superconformally covariant structures from the building blocks $\left(X_{j k+}, \mathfrak{X}_{i+}, \Theta_{i}, \lambda_{i}\right)$ - these are the fermionic analogues of $P, S, Q$. We define them below and also give there transformation under superinversion.

\footnotetext{
${ }^{16}$ Note that the $S_{k}$ in [17] has an extra factor of $i P_{k}$ compared to ours.
} 
a) Fermionic analogues of $\boldsymbol{P}_{\boldsymbol{i}}$. Define

$$
\pi_{i j}=\lambda_{i} X_{i j+} \Theta_{j}
$$

Then under superinversion

$$
\pi_{i j} \rightarrow-\lambda_{i} X_{i-}^{-1} X_{i+}^{-1} X_{i j+} X_{j-}^{-1} X_{j-} \Theta_{j}=-\frac{1}{x_{i}^{2}} \pi_{i j}
$$

Similarly,

$$
\Pi_{i j}=\Theta_{i} X_{i j+} \Theta_{j}, \quad \Pi_{i j} \rightarrow \Pi_{i j}
$$

It turns out, however, that

$$
\Pi_{i j}=0
$$

b) Fermionic analogues of $S_{i}$.

$$
\begin{array}{rlrl}
\sigma_{13} & =\frac{\lambda_{1} X_{12+} X_{23+} \Theta_{3}}{\tilde{X}_{12} \tilde{X}_{23} \tilde{X}_{31}}, & \sigma_{13} \rightarrow x_{3}^{2} \sigma_{13} \\
\Sigma_{13}=\frac{\Theta_{1} X_{12+} X_{23+} \Theta_{3}}{\tilde{X}_{12} \tilde{X}_{23} \tilde{X}_{31}}, & \Sigma_{13} \rightarrow-x_{1}^{2} x_{3}^{2} \Sigma_{13}
\end{array}
$$

$\sigma_{32}, \sigma_{21}, \Sigma_{32}, \Sigma_{21}$ are similarly defined through cyclic permutation of the indices. It follows that

$$
\tilde{X}_{i j}^{2} \Sigma_{i j} \rightarrow-\tilde{X}_{i j}^{2} \Sigma_{i j}
$$

c) Fermionic analogues of $Q_{i}$.

$$
\begin{array}{rlrl}
\omega_{i} & =\lambda_{i} \mathfrak{X}_{i+} \Theta_{i}, & \omega_{i} & \rightarrow-x_{i}^{2} \omega_{i} \\
\Omega_{i} & =\Theta_{i} \mathfrak{X}_{i+} \Theta_{i}, & \Omega_{i} \rightarrow x_{i}^{4} \Omega_{i}
\end{array}
$$

However, $\Omega_{i}$ is identically zero

$$
\Omega_{i}=0
$$

The invariants constructed out of the product of two parity odd (or two parity even) covariant structures would be parity even, and since we have already listed all the parity even invariants, would be expressible in terms of $P_{i}, Q_{i}, R_{i}$. Thus, we find the following relations for the above covariant structures

$$
\begin{aligned}
\pi_{i j}^{2} & =\sigma_{i j}^{2}=\omega_{i}^{2}=0 & & \\
\pi_{i j} \omega_{i} & =0 & & \\
\frac{1}{\tilde{X}_{12}^{2}} \pi_{12} \pi_{23} & =-R_{1} R_{2}, & & \frac{1}{\tilde{X}_{23}^{2}} \pi_{23} \pi_{31}=-R_{2} R_{3}, \quad \frac{1}{\tilde{X}_{31}^{2}} \pi_{31} \pi_{12}=-R_{3} R_{1} \\
\frac{1}{\tilde{X}_{i j}^{2}} \pi_{i j} \pi_{j i} & =R_{i} R_{j}=\tilde{X}_{i j}^{2} \sigma_{i j} \sigma_{j i} & & \\
\tilde{X}_{12}^{2} \sigma_{21} \sigma_{32} & =R_{2} R_{3}, & \tilde{X}_{23}^{2} \sigma_{32} \sigma_{13}=R_{3} R_{1}, & \tilde{X}_{31}^{2} \sigma_{13} \sigma_{21}=R_{1} R_{2} \\
\tilde{X}_{i j}^{2} \omega_{i} \omega_{j} & =-R_{i} R_{j} & &
\end{aligned}
$$


From the above covariant structures it is possible to build additional parity odd fermionic invariants by taking products of a parity even and a parity odd covariant structure. ${ }^{17}$ Thus, we have

$$
T_{i j}=\pi_{i j} \sigma_{j i}
$$

and under superinversion

$$
T_{i j} \rightarrow-T_{i j}
$$

Note that $\pi_{i j} \neq \pi_{j i}$ so $\left\{\pi_{12}, \pi_{23}, \pi_{31}\right\}$ is a different set of parity odd covariant structures than $\left\{\pi_{21}, \pi_{32}, \pi_{13}\right\}$ (the same is true for the even structures $\sigma_{i j}$ ). However, because the following relation is true

$$
T_{i j}=-T_{j i}
$$

it follows that we have only three odd invariant structures:

$$
T_{1} \equiv T_{23}=\pi_{23} \sigma_{32}, \quad T_{2} \equiv T_{31}=\pi_{31} \sigma_{13}, \quad T_{3} \equiv T_{12}=\pi_{12} \sigma_{21}
$$

We may also define

$$
T_{23}^{\prime}=\pi_{12} \sigma_{31}, \quad T_{31}^{\prime}=\pi_{23} \sigma_{12}, \quad T_{12}^{\prime}=\pi_{31} \sigma_{23} \quad T_{i j}^{\prime} \rightarrow-T_{i j}^{\prime}
$$

with $T_{32}^{\prime}=\pi_{13} \sigma_{21}, \quad T_{13}^{\prime}=\pi_{21} \sigma_{32}, \quad T_{21}^{\prime}=\pi_{32} \sigma_{13}$ again being related to the above by

$$
P_{3} T_{21}^{\prime}=-P_{2} T_{31}^{\prime}, \quad P_{1} T_{32}^{\prime}=-P_{3} T_{12}^{\prime}, \quad P_{2} T_{13}^{\prime}=-P_{1} T_{23}^{\prime}
$$

Also

$$
\bar{T}_{i j}=\tilde{X}_{i j}^{2} \sigma_{j i} \omega_{j}, \quad \bar{T}_{i j} \rightarrow-\bar{T}_{i j}
$$

Again, we have the relation

$$
\bar{T}_{i j} Q_{i}=\bar{T}_{j i} Q_{j}
$$

thus we have only three $\bar{T}_{i j}$ 's.

Likewise, we have

$$
\hat{T}_{12}=\tilde{X}_{12}^{2} \sigma_{31} \omega_{2}, \hat{T}_{23}=\tilde{X}_{23}^{2} \sigma_{12} \omega_{3} \hat{T}_{31}=\tilde{X}_{31}^{2} \sigma_{23} \omega_{1} \quad \hat{T}_{i j} \rightarrow-\hat{T}_{i j}
$$

with $\hat{T}_{21}, \hat{T}_{32}, \hat{T}_{13}$ being related to the above by

$$
P_{j} \hat{T}_{i j}=P_{i} \hat{T}_{j i}
$$

We also have the following relations involving $\Sigma_{i j}$

$$
\Sigma_{i j}=\Sigma_{j i}, \quad \tilde{X}_{12}^{2} \Sigma_{12}=\tilde{X}_{23}^{2} \Sigma_{32}=\tilde{X}_{31}^{2} \Sigma_{31}
$$

Therefore, here we get just one parity odd invariant

$$
T \equiv \tilde{X}_{i j}^{2} \Sigma_{i j}
$$

\footnotetext{
${ }^{17}$ Note that structures like $x_{i} \omega_{i}, \pi_{i j} / x_{i}$ would be parity odd invariants under inversion. However, these are not Poincare invariant (since correlation functions should depend only on differences $\left(x_{i j}\right)$ of the coordinates). We could also construct structures like $U=\tilde{X}_{12} \tilde{X}_{23} \tilde{X}_{31} \omega_{1} \omega_{2} \omega_{3}$ which would be an odd invariant $(U \rightarrow-U)$. However, it is identically zero because the product of three different $\Theta$ 's vanishes.
} 
It turns out that $T_{i j}^{\prime}, \bar{T}_{i j}, \hat{T}_{i j}, \tilde{X}_{i j}^{2} \Sigma_{i j}$ can be expressed in terms of $T_{i}$ by means of the following relations

$$
\begin{aligned}
& P_{1} T_{31}^{\prime}=P_{3} T_{1}, \\
& P_{2} T_{12}^{\prime}=P_{1} T_{2}, \\
& P_{3} T_{23}^{\prime}=P_{2} T_{3} \\
& P_{3} \bar{T}_{12}=-Q_{2} T_{3} \text {, } \\
& P_{1} \bar{T}_{23}=-Q_{3} T_{1}, \\
& P_{2} \bar{T}_{31}=-Q_{1} T_{2} \\
& \frac{1}{2} P_{2} \tilde{X}_{13}^{2} \Sigma_{13}=T_{2} \text {, } \\
& P_{1} \hat{T}_{23}=-P_{2} T_{1}, \\
& \frac{1}{2} P_{3} \tilde{X}_{21}^{2} \Sigma_{21}=T_{3} \text {, } \\
& \frac{1}{2} P_{1} \tilde{X}_{32}^{2} \Sigma_{32}=T_{1} \\
& P_{2} \hat{T}_{31}=-P_{3} T_{2}, \\
& P_{3} \hat{T}_{12}=-P_{1} T_{3}
\end{aligned}
$$

Making use of the above equation and eq. (7.36) we can express all parity odd fermionic structures in terms of $T$

$$
\begin{aligned}
& T_{2}=\frac{1}{2} P_{2} T, \quad T_{3}=\frac{1}{2} P_{3} T, \quad T_{1}=\frac{1}{2} P_{1} T \\
& \bar{T}_{12}=-\frac{1}{2} Q_{2} T, \quad \bar{T}_{23}=-\frac{1}{2} Q_{3} T, \quad \bar{T}_{31}=-\frac{1}{2} Q_{1} T \\
& T_{31}^{\prime}=\frac{1}{2} P_{3} T, \quad T_{12}^{\prime}=\frac{1}{2} P_{1} T, \quad T_{23}^{\prime}=\frac{1}{2} P_{2} T \\
& \hat{T}_{12}=-\frac{1}{2} P_{1} T, \quad \hat{T}_{23}=-\frac{1}{2} P_{2} T, \quad \hat{T}_{31}=-\frac{1}{2} P_{3} T
\end{aligned}
$$

To summarize, from our fermionic covariant structures we could construct five parity odd invariants $T_{i}, T_{i j}^{\prime}, \bar{T}_{i j}, \hat{T}_{i j}, T$. However, only $T$ suffices as the other four are related to it through the above simple relations.

Summary of this section: we have thus obtained the superconformal invariants $P_{i}, Q_{i}, R_{i}, S_{i}, T$ (listed in tabular form at the beginning of this section) out of which the invariant structures for particular 3-point functions can be constructed as monomials in these variables. Before we do this, however, we need to determine all the relations between these variables using which we can get a linearly independent basis of monomial structures for 3-point functions.

\subsection{Relations between the invariant structures}

Since the $\mathcal{N}=1$ superconformal group in 3 dimensions has 14 generators (10 bosonic, 4 fermionic), out of $\left(x_{i}, \theta_{i}, \lambda_{i}\right)(i=1,2,3)$ we can construct $7 \times 3-14=7$ superconformal invariants. Thus among the nine parity even structures $\left(P_{i}, Q_{i}, R_{i}\right)$ we must have two relations. One of them is the supersymmetrized version of the non-linear relation (2.14) in [17]

$P_{1}^{2} Q_{1}+P_{2}^{2} Q_{2}+P_{3}^{2} Q_{3}-2 P_{1} P_{2} P_{3}-Q_{1} Q_{2} Q_{3}-\frac{i}{2}\left(R_{1} R_{2} P_{3} Q_{3}+R_{2} R_{3} P_{1} Q_{1}+R_{3} R_{1} P_{2} Q_{2}\right)=0$

This cuts down the number of independent invariants by one. We also have the following triplet of relations which vanishes identically when the Grassmann variables are set to zero (fermionic relations) and reduces the number of invariants to seven:

$$
\begin{aligned}
& P_{2} R_{1} R_{2}+Q_{1} R_{2} R_{3}+P_{3} R_{3} R_{1}=0 \\
& P_{3} R_{2} R_{3}+Q_{2} R_{3} R_{1}+P_{1} R_{1} R_{2}=0 \\
& P_{1} R_{3} R_{1}+Q_{3} R_{1} R_{2}+P_{2} R_{2} R_{3}=0
\end{aligned}
$$


There are further non-linear relations involving the $S$ 's. Since the squares or products of $S$ 's are parity even, we expect them to be determined in terms of the parity even structures. Indeed, we find

$$
\begin{aligned}
& S_{1}^{2}=P_{1}^{2}-Q_{2} Q_{3}-i P_{1} R_{2} R_{3}, \quad S_{2}^{2}=P_{2}^{2}-Q_{3} Q_{1}-i P_{2} R_{3} R_{1}, \quad S_{3}^{2}=P_{3}^{2}-Q_{1} Q_{2}-i P_{3} R_{1} R_{2} \\
& S_{1} S_{2}=P_{3} Q_{3}-P_{1} P_{2}, \quad S_{2} S_{3}=P_{1} Q_{1}-P_{2} P_{3}, \quad S_{3} S_{1}=P_{2} Q_{2}-P_{3} P_{1}
\end{aligned}
$$

They imply that the most general odd structures that can occur in any three point function are linear in $S_{i}$. It turns out there exist further linear relations between the parity odd structures. We find the following basic linear relationships between the various parity odd invariant structures:

at $O\left(\lambda_{1} \lambda_{2} \lambda_{3}\right)$ :

$$
R_{1} S_{1}+R_{2} S_{2}+R_{3} S_{3}=0
$$

At $O\left(\lambda_{1}^{2} \lambda_{2} \lambda_{3}, \lambda_{1} \lambda_{2}^{2} \lambda_{3}, \lambda_{1} \lambda_{2} \lambda_{3}^{2}\right)$ :

$$
\begin{aligned}
Q_{1} S_{1}+P_{2} S_{3}+P_{3} S_{2}-\frac{i}{2} P_{2} P_{3} T & =0 \\
Q_{2} S_{2}+P_{3} S_{1}+P_{1} S_{3}-\frac{i}{2} P_{1} P_{3} T & =0 \\
Q_{3} S_{3}+P_{1} S_{2}+P_{2} S_{1}-\frac{i}{2} P_{1} P_{2} T & =0
\end{aligned}
$$

and

$$
\begin{aligned}
& S_{2} R_{1} R_{2}+S_{3} R_{3} R_{1}+T\left(Q_{1} P_{1}-P_{2} P_{3}\right)=0 \\
& S_{3} R_{2} R_{3}+S_{1} R_{1} R_{2}+T\left(Q_{2} P_{2}-P_{3} P_{1}\right)=0 \\
& S_{1} R_{3} R_{1}+S_{2} R_{2} R_{3}+T\left(Q_{3} P_{3}-P_{1} P_{2}\right)=0
\end{aligned}
$$

From eq. (7.45) follows:

$$
\begin{aligned}
& S_{2} R_{1} R_{2}-S_{3} R_{3} R_{1}=0 \\
& S_{3} R_{2} R_{3}-S_{1} R_{1} R_{2}=0 \\
& S_{1} R_{3} R_{1}-S_{2} R_{2} R_{3}=0
\end{aligned}
$$

From these follow other linear relations at higher orders in $\lambda_{1}, \lambda_{2}, \lambda_{3}$ :

$$
\begin{aligned}
& Q_{1} P_{1} S_{1}+Q_{2} P_{2} S_{2}-Q_{3} P_{3} S_{3}+2 P_{1} P_{2} S_{3}-\frac{i}{2} T P_{1} P_{2} P_{3}=0 \\
& Q_{2} P_{2} S_{2}+Q_{3} P_{3} S_{3}-Q_{1} P_{1} S_{1}+2 P_{2} P_{3} S_{1}-\frac{i}{2} T P_{1} P_{2} P_{3}=0 \\
& Q_{3} P_{3} S_{3}+Q_{1} P_{1} S_{1}-Q_{2} P_{2} S_{2}+2 P_{3} P_{1} S_{2}-\frac{i}{2} T P_{1} P_{2} P_{3}=0
\end{aligned}
$$

Adding the above equations gives

$$
Q_{1} P_{1} S_{1}+Q_{2} P_{2} S_{2}+Q_{3} P_{3} S_{3}-\frac{3 i}{2} T P_{1} P_{2} P_{3}+2\left(P_{1} P_{2} S_{3}+P_{2} P_{3} S_{1}+P_{3} P_{1} S_{2}\right)=0
$$

Also, we get

$$
R_{1} R_{2}\left(S_{1} P_{2}+\frac{1}{2} Q_{3} S_{3}\right)+R_{2} R_{3}\left(S_{2} P_{3}+\frac{1}{2} Q_{1} S_{1}\right)+R_{3} R_{1}\left(S_{3} P_{1}+\frac{1}{2} Q_{2} S_{2}\right)=0
$$




$$
\begin{aligned}
& \left(P_{1}^{2} Q_{1}-P_{2}^{2} Q_{2}\right) P_{3} S_{3}+\left(P_{3}^{2}-Q_{1} Q_{2}-i P_{3} R_{1} R_{2}\right)\left(Q_{1} P_{1} S_{1}-Q_{2} P_{2} S_{2}\right)=0 \\
& \left(P_{2}^{2} Q_{2}-P_{3}^{2} Q_{3}\right) P_{1} S_{1}+\left(P_{1}^{2}-Q_{2} Q_{3}-i P_{1} R_{2} R_{3}\right)\left(Q_{2} P_{2} S_{2}-Q_{3} P_{3} S_{3}\right)=0 \\
& \left(P_{3}^{2} Q_{3}-P_{1}^{2} Q_{1}\right) P_{2} S_{2}+\left(P_{2}^{2}-Q_{3} Q_{1}-i P_{2} R_{3} R_{1}\right)\left(Q_{3} P_{3} S_{3}-Q_{1} P_{1} S_{1}\right)=0
\end{aligned}
$$

and so on. All these relations can be put to use in eliminating linearly dependent structures in 3-point functions. The above relations between the invariant structures extend the corresponding non-supersymmetric ones in [17].

We also have the following relations

$$
T^{2}=0, \quad F T=0, \quad S_{i} T=-\epsilon_{i j k} R_{j} R_{k} \quad \text { sum over } j, k
$$

where $F$ stands for any of the fermionic covariant/invariant structures. This implies that for any 3-point function it suffices to consider parity odd structures linear in $T, S_{i}$. Thus $S_{i}, T$ comprise all the parity odd invariants we need in writing down possible odd structures in the 3-point functions of higher spin operators and we need only terms linear in these invariants.

\subsection{Simple examples of three point functions}

\subsubsection{Independent invariant structures for three point functions}

Below we write down the possible superconformal invariant structures that can occur in specific three point functions $\left\langle J_{s_{1}}(1) J_{s_{2}}(2) J_{s_{3}}(3)\right\rangle$. We consider the case of abelian currents so that, when some spins are equal, the correlator is (anti-) symmetric under pairwise exchanges of identical currents. We use only superconformal invariance to constrain the correlators, so the results of this section apply even if the higher spin symmetry is broken (that is, if $J_{s}$ is not conserved for $s>2$ ). All that is required is that $J_{s}$ are higher spin operators transforming suitably under superconformal transformations. ${ }^{18}$

Under the pairwise exchange $2 \leftrightarrow 3$ we have

$$
A_{1} \rightarrow-A_{1}, \quad A_{2} \rightarrow-A_{3}, \quad A_{3} \rightarrow-A_{2}, \quad T \rightarrow T
$$

where $A$ stands for any of $P, Q, R, S$.

$\left\langle\boldsymbol{J}_{\frac{1}{2}} \boldsymbol{J}_{\frac{1}{2}} \boldsymbol{J}_{0}\right\rangle:$ here $J_{0}$ is a scalar operator with $\Delta=1$. It is clear that any term that can occur is of order $\lambda_{1} \lambda_{2}$. Thus the possible structures that can occur in this correlator are:

$$
P_{3}, R_{1} R_{2}, S_{3}, P_{3} T
$$

We also computed this correlator explicitly in the free field theory (like the $\left\langle J_{\frac{1}{2}} J_{\frac{1}{2}}\right\rangle$ correlator in the previous section) and the result is (with $\Delta_{1}=\Delta_{2}=\frac{3}{2}, \Delta_{3}=\frac{1}{2}$ ):

$$
\frac{1}{\tilde{X}_{12} \tilde{X}_{23} \tilde{X}_{31}}\left(P_{3}-\frac{i}{2} R_{1} R_{2}\right)
$$

The odd piece can not occur in the free field case.

\footnotetext{
${ }^{18}$ We take $J_{\alpha_{1} \alpha_{2} \ldots \ldots \alpha_{s_{i}}}$ to be a primary with arbitrary conformal dimension $\Delta_{i}$ so that $J_{s_{i}} \equiv$ $\lambda^{\alpha_{1}} \lambda^{\alpha_{2}} \ldots \lambda^{\alpha_{s_{i}}} J_{\alpha_{1} \alpha_{2} \ldots \ldots \alpha_{s_{i}}}$ has dimension $\Delta_{i}-s_{i}$. In general $J_{s_{i}}$ need not be conserved. However, if the unitarity bound is attained $-\Delta_{i}=s_{i}+1$ for $s_{i} \geq \frac{1}{2} ; \Delta_{i}=\frac{1}{2}$ for $s_{i}=0$ - then $J_{s_{i}}$, being a short primary, is necessarily conserved: $D_{(i) \alpha} \frac{\partial}{\partial \lambda_{(i) \alpha}} J_{s_{i}}=0$.
} 
$\left\langle\boldsymbol{J}_{\frac{1}{2}} \boldsymbol{J}_{\frac{1}{2}} \boldsymbol{J}_{\frac{1}{2}}\right\rangle$ : note that this has to be antisymmetric under exchange of any two currents. However the only two possible structures $\sum R_{i} P_{i}, \sum R_{i} S_{i}$ are symmetric under this exchange. Thus $\left\langle J_{\frac{1}{2}} J_{\frac{1}{2}} J_{\frac{1}{2}}\right\rangle$ vanishes.

$\left\langle\boldsymbol{J}_{\boldsymbol{s}} \boldsymbol{J}_{\mathbf{0}} \boldsymbol{J}_{\mathbf{0}}\right\rangle:$ for $s$ an even integer, the correlator is

$$
\left\langle J_{s} J_{0} J_{0}\right\rangle=\frac{1}{\tilde{X}_{12} \tilde{X}_{23} \tilde{X}_{31}} Q_{1}^{s}
$$

In this case no other structure can occur. For s odd or half-integral, the correlator is zero.

$\left\langle\boldsymbol{J}_{\boldsymbol{s}} \boldsymbol{J}_{\frac{1}{2}} \boldsymbol{J}_{\frac{1}{2}}\right\rangle:$ for $s$ an even integer, the possible structures are

$$
\begin{array}{lll}
Q_{1}^{s} P_{1}, & Q_{1}^{s-1} P_{2} P_{3}, & R_{2} R_{3} Q_{1}^{s}, \\
Q_{1}^{s-1}\left(P_{2} S_{3}+P_{3} S_{2}\right), & Q_{1}^{s} P_{1} T, & Q_{1}^{s-1} P_{2} P_{3} T
\end{array}
$$

The structure $R_{1} Q_{1}^{s-1}\left(R_{2} P_{2}-R_{3} P_{3}\right)$ is also possible but using eq. (7.43) equals $-R_{2} R_{3} Q_{1}^{s}$ and hence can be eliminated while writing down independent superconformal invariant structures. Similarly, the structure $Q_{1}^{s} S_{1}$ can be written in terms of others listed above by using eq. (7.46) and $R_{1} Q_{1}^{s-1}\left(R_{2} S_{2}-R_{3} S_{3}\right)$ in terms of the last two structures above by using eq. (7.47)

For $s$ odd, antisymmetry under the exchange $2 \leftrightarrow 3$ allows only the following possible structures

$$
R_{1} Q_{1}^{s-1}\left(R_{2} P_{2}+R_{3} P_{3}\right), Q_{1}^{s-1}\left(P_{2} S_{3}-P_{3} S_{2}\right)
$$

The structure $R_{1} Q_{1}^{s-1}\left(R_{2} S_{2}+R_{3} S_{3}\right)$ vanishes on using eq. (7.45).

$\left\langle J_{1} J_{1} J_{0}\right\rangle:$ the possible structures are

$$
Q_{1} Q_{2}, P_{3}^{2}, R_{1} R_{2} P_{3}, R_{1} R_{2} S_{3}, P_{3} S_{3}, Q_{1} Q_{2} T, P_{3}^{2} T
$$

$\left\langle\boldsymbol{J}_{\mathbf{1}} \boldsymbol{J}_{\mathbf{1}} \boldsymbol{J}_{\mathbf{1}}\right\rangle:$ note that all the parity even structures that can occur in $\left\langle J_{1} J_{1} J_{1}\right\rangle$ are those that are present in the non-linear relation eq. (7.42) but all these structures are antisymmetric under the exchange of any two currents whereas this correlator is symmetric under the same exchange. Hence the parity even part of $\left\langle J_{1} J_{1} J_{1}\right\rangle$ vanishes. For the same reason no possible parity odd structures can occur either. Thus $\left\langle J_{1} J_{1} J_{1}\right\rangle$ vanishes in general.

$\left\langle\boldsymbol{J}_{\frac{3}{2}} \boldsymbol{J}_{\frac{1}{2}} \boldsymbol{J}_{0}\right\rangle:$ here the possible structures are

$$
Q_{1} P_{3}, R_{1} R_{2} Q_{1}, Q_{1} S_{3}, Q_{1} P_{3} T
$$

$\left\langle\boldsymbol{J}_{\frac{3}{2}} \boldsymbol{J}_{\frac{1}{2}} \boldsymbol{J}_{\frac{1}{2}}\right\rangle:$ the linearly independent structures are

$$
R_{1} Q_{1} P_{1}, R_{1} P_{2} P_{3}, Q_{1}\left(R_{2} P_{2}+R_{3} P_{3}\right), R_{1} Q_{1} S_{1}
$$

Two other possible fermionic parity odd structures can be eliminated using eqs. (7.45), (7.46). 
$\left\langle\boldsymbol{J}_{\frac{3}{2}} \boldsymbol{J}_{\frac{1}{2}} \boldsymbol{J}_{1}\right\rangle:$ after eliminating some structures using the relations in section 7.2 we get the following linearly independent structures:

$$
\begin{aligned}
& Q_{1} Q_{2} P_{2}, \quad Q_{1} P_{1} P_{3}, \quad P_{3}^{2} P_{2}, \quad R_{1} R_{2} Q_{1} P_{1}, \quad R_{1} R_{2} P_{2} P_{3}, \quad R_{3} R_{1} Q_{1} Q_{2}, \\
& Q_{1} P_{1} S_{3}, \quad Q_{1} P_{3} S_{1}, \quad P_{2} P_{3} S_{3}, \quad R_{1} R_{2} P_{2} S_{3}, \quad Q_{1} Q_{2} P_{2} T, \quad Q_{1} P_{1} P_{3} T, \quad P_{3}^{2} P_{2} T \\
& \left\langle J_{\frac{3}{2}} J_{\frac{3}{2}} J_{\frac{3}{2}}\right\rangle: \\
& Q_{1} Q_{2} Q_{3} \sum_{i} R_{i} P_{i}, \quad \sum_{\text {cyclic }} R_{1} Q_{2} Q_{3} P_{2} P_{3}, \quad \sum_{i} R_{i} Q_{i} P_{i}^{3}, \quad P_{1} P_{2} P_{3} \sum_{i} R_{i} P_{i}, \\
& \sum_{i} R_{i} Q_{i} P_{i}^{2} S_{i}
\end{aligned}
$$

The structure $\sum_{c y c} R_{1} P_{1}\left(P_{2}^{2} Q_{2}+P_{3}^{2} Q_{3}\right)$ can, by using the non-linear identity eq. (7.42), be expressed in terms of the above structures and hence need not be included. The structure $\sum_{\text {cyclic }} R_{1} Q_{2} Q_{3}\left(P_{2} S_{3}+P_{3} S_{2}\right)$ vanishes on using eqs. (7.46), (7.45)

$\left\langle\boldsymbol{J}_{2} \boldsymbol{J}_{1} \boldsymbol{J}_{1}\right\rangle:$ the possible linearly independent structures are

$$
\begin{array}{llll}
Q_{1}^{2} Q_{2} Q_{3}, & Q_{1}^{2} P_{1}^{2}, & Q_{1} P_{1} P_{2} P_{3}, & P_{2}^{2} P_{3}^{2}, \\
R_{2} R_{3} P_{1} Q_{1}^{2}, & R_{2} R_{3} P_{2} P_{3} Q_{1}, & & \\
Q_{1} Q_{2} P_{2} S_{2}+Q_{1} Q_{3} P_{3} S_{3}, & P_{2}^{2} P_{3} S_{3}+P_{3}^{2} P_{2} S_{2}, & & \\
R_{1} R_{2} P_{2}^{2} S_{3}+R_{3} R_{1} P_{3}^{2} S_{2}, & & & \\
Q_{1}^{2} Q_{2} Q_{3} T, & Q_{1}^{2} P_{1}^{2} T, & Q_{1} P_{1} P_{2} P_{3} T, & P_{2}^{2} P_{3}^{2} T
\end{array}
$$

Other structures are possible, but can be written in terms of the other structures listed above by using the relations in section 7.2.

$\left\langle\boldsymbol{J}_{3} \boldsymbol{J}_{1} \boldsymbol{J}_{1}\right\rangle:$ as before, after eliminating some structures which are antisymmetric under the exchange $2 \leftrightarrow 3$ we are left with the following linearly independent basis for $\left\langle J_{3} J_{1} J_{1}\right\rangle$ :

$$
\begin{array}{ll}
Q_{1}^{2}\left(P_{2}^{2} Q_{2}-P_{3}^{2} Q_{3}\right), & \\
Q_{1}^{2}\left(R_{1} R_{2} P_{1} P_{2}-R_{3} R_{1} P_{3} P_{1}\right), & Q_{1}\left(R_{1} R_{2} P_{2}^{2} P_{3}-R_{3} R_{1} P_{3}^{2} P_{2}\right), \\
Q_{1}^{2}\left(P_{2} Q_{2} S_{2}-P_{3} Q_{3} S_{3}\right), & Q_{1}\left(P_{3}^{2} P_{2} S_{2}-P_{2}^{2} P_{3} S_{3}\right), \\
Q_{1}\left(R_{1} R_{2} P_{2}^{2} S_{3}-R_{3} R_{1} P_{3}^{2} S_{2}\right), & Q_{1}^{2}\left(P_{2}^{2} Q_{2}-P_{3}^{2} Q_{3}\right) T
\end{array}
$$

Again, linearly dependent structures have been eliminated using the relations of section 7.2.

$\left\langle\boldsymbol{J}_{4} \boldsymbol{J}_{1} \boldsymbol{J}_{1}\right\rangle$ : the structures that occur here are the same as $Q_{1}^{2}$ times the structures in $\left\langle J_{2} J_{1} J_{1}\right\rangle$.

$\left\langle\boldsymbol{J}_{\boldsymbol{s}} \boldsymbol{J}_{\mathbf{1}} \boldsymbol{J}_{\mathbf{1}}\right\rangle$ : for $s$ even this again equals $Q_{1}^{s-2}\left\langle J_{2} J_{1} J_{1}\right\rangle$ (this was noted, for the nonsupersymmetric case, in ref. [17]- it continues to hold in our case). For $s$ odd and greater than three this correlator equals $Q_{1}^{s-2}\left\langle J_{3} J_{1} J_{1}\right\rangle$. Thus the number of possible tensor structures in $\left\langle J_{s} J_{1} J_{1}\right\rangle$ does not increase with $s$. 
$\left\langle\boldsymbol{J}_{2} \boldsymbol{J}_{2} \boldsymbol{J}_{\mathbf{2}}\right\rangle:$ the following are the possible independent invariant structures

$$
\begin{aligned}
& Q_{1}^{2} Q_{2}^{2} Q_{3}^{2}, \quad P_{1}^{2} P_{2}^{2} P_{3}^{2}, \quad Q_{1} Q_{2} Q_{3} P_{1} P_{2} P_{3}, \quad \sum_{i} Q_{i}^{2} P_{i}^{4}, \\
& Q_{1} Q_{2} Q_{3} \sum_{\text {cyclic }} Q_{3} P_{3} R_{1} R_{2}, \quad P_{1} P_{2} P_{3} \sum_{\text {cyclic }} Q_{3} P_{3} R_{1} R_{2}, \\
& P_{1} P_{2} P_{3} \sum_{\text {cyclic }} P_{1} P_{2} S_{3}, \quad \sum_{i} Q_{i}^{2} P_{i}^{3} S_{i} \\
& Q_{1} Q_{2} Q_{3} \sum_{\text {cyclic }} Q_{3} S_{3} R_{1} R_{2}, \quad P_{1} P_{2} P_{3} \sum_{\text {cyclic }} Q_{3} S_{3} R_{1} R_{2}, \\
& Q_{1}^{2} Q_{2}^{2} Q_{3}^{2} T, \quad P_{1}^{2} P_{2}^{2} P_{3}^{2} T, \quad Q_{1} Q_{2} Q_{3} P_{1} P_{2} P_{3} T, \quad \sum_{i} Q_{i}^{2} P_{i}^{4} T
\end{aligned}
$$

Many other linearly dependent structures have been eliminated using the relations in section 7.2.

As is evident, the number of invariant structures needed to construct the 3-point correlator increases rapidly as the spins of the operators increase and we will not consider more examples.

It is clear from the above examples that the general structure of the 3-point function is the following:

$$
\left\langle J_{s_{1}} J_{s_{2}} J_{s_{3}}\right\rangle=\frac{1}{\tilde{X}_{12}^{m_{123}} \tilde{X}_{23}^{m_{231}} \tilde{X}_{31}^{m_{312}}} \sum_{n} \mathcal{F}_{n}\left(P_{i}, Q_{i}, R_{i}, S_{i}, T\right)
$$

where $m_{i j k} \equiv\left(\Delta_{i}-s_{i}\right)+\left(\Delta_{j}-s_{j}\right)-\left(\Delta_{k}-s_{k}\right)$ and the sum is over all the independent invariant structures $\mathcal{F}_{n}$, each of homogeneity $\lambda_{1}^{2 s_{1}} \lambda_{2}^{2 s_{2}} \lambda_{3}^{2 s_{3}}$. Since the 3-point function is linear in the parity odd invariants and linear or bilinear in the $R$ 's (either $R_{i}$ or $R_{j} R_{k}, j \neq$ $k$ ), we have the following structure for $\mathcal{F}_{n}$ :

$$
\begin{aligned}
\mathcal{F}_{n}= & F_{n}^{(1)}\left(P_{i}, Q_{i}\right)+a_{n}^{(1)} F_{n}^{(1)}\left(P_{i}, Q_{i}\right) T+a_{n}^{(2)} F_{n}^{(2)}\left(P_{i}, Q_{i}\right) S_{i}+a_{n}^{(3)} F_{n}^{(3)}\left(P_{i}, Q_{i}\right) R_{i} \\
& +a_{n}^{(4)} F_{n}^{(4)}\left(P_{i}, Q_{i}\right) R_{i} S_{j}+a_{n}^{(5)} F_{n}^{(5)}\left(P_{i}, Q_{i}\right) R_{j} R_{k}+a_{n}^{(6)} F_{n}^{(6)}\left(P_{i}, Q_{i}\right) R_{j} R_{k} S_{l}
\end{aligned}
$$

Here each $F_{n}^{(a)}\left(P_{i}, Q_{i}\right)$ is a monomial in $P$ 's and $Q$ 's such that each term on the r.h.s. above has homogeneity $\lambda_{1}^{2 s_{1}} \lambda_{2}^{2 s_{2}} \lambda_{3}^{2 s_{3}}{ }^{19}$

\subsubsection{Three point functions of conserved currents}

We have so far considered the constraints on the structure of the three-point functions of higher spin operators arising due to superconformal invariance alone. We will now see how the structure is further constrained by current conservation, i.e, when the operators are actually conserved higher spin currents. In this section we present evidence for the claim

\footnotetext{
${ }^{19}$ The $\operatorname{six} F_{n}^{(a)}\left(P_{i}, Q_{i}\right)$ are not independent functions. $F_{n}^{(2)}, F_{n}^{(4)}, F_{n}^{(6)}$ can be obtained from $F_{n}^{(1)}, F_{n}^{(3)}, F_{n}^{(5)}$, respectively, by replacing a $P_{i}^{p}$ in the latter by $P_{i}^{p-1} S_{i}$ (suitably (anti-)symmetrized if some spins are equal in the 3 -point function).
} 


\begin{tabular}{|c|c|c|}
\hline Three-pt function & Even & Odd \\
\hline$\left\langle J_{\frac{1}{2}} J_{\frac{1}{2}} J_{0}\right\rangle$ & $P_{3}-\frac{i}{2} R_{1} R_{2}$ & $S_{3}-\frac{i}{2} P_{3} T$ \\
\hline$\left\langle J_{1} J_{\frac{1}{2}} J_{0}\right\rangle$ & $P_{3} R_{1}+\frac{1}{2} Q_{1} R_{2}$ & 0 \\
\hline$\left\langle J_{1} J_{1} J_{0}\right\rangle$ & $\frac{1}{2} Q_{1} Q_{2}+P_{3}^{2}-i R_{1} R_{2} P_{3}$ & $S_{3} P_{3}+\frac{i}{2}\left(S_{3} R_{1} R_{2}-Q_{1} Q_{2} T\right)$ \\
\hline$\left\langle J_{\frac{3}{2}} J_{\frac{1}{2}} J_{0}\right\rangle$ & $P_{3} Q_{1}-\frac{i}{2} Q_{1} R_{1} R_{2}$ & $Q_{1} S_{3}-i Q_{1} P_{3} T$ \\
\hline$\left\langle J_{\frac{3}{2}} J_{\frac{1}{2}} J_{\frac{1}{2}}\right\rangle$ & $Q_{1} R_{1} P_{1}+Q_{1}\left(R_{2} P_{2}+R_{3} P_{3}\right)+2 R_{1} P_{2} P_{3}$ & 0 \\
\hline$\left\langle J_{2} J_{\frac{1}{2}} J_{\frac{1}{2}}\right\rangle$ & $Q_{1}^{2} P_{1}-4 Q_{1} P_{2} P_{3}-\frac{5 i}{2} R_{2} R_{3} Q_{1}^{2}$ & $Q_{1}\left(P_{2} S_{3}+P_{3} S_{2}\right)$ \\
& & $+\frac{i}{2}\left(Q_{1}^{2} P_{1}-3 Q_{1} P_{2} P_{3}\right) T$ \\
\hline
\end{tabular}

Table 2. Explicit examples of conserved three-point functions.

that the three point function of the conserved higher spin currents in $\mathcal{N}=1$ superconformal field theory consists of two linearly independent parts, i.e.,

$$
\left\langle J_{s_{1}} J_{s_{2}} J_{s_{3}}\right\rangle=\frac{1}{\tilde{X}_{12} \tilde{X}_{23} \tilde{X}_{31}}\left(a\left\langle J_{s_{1}} J_{s_{2}} J_{s_{3}}\right\rangle \text { even }+b\left\langle J_{s_{1}} J_{s_{2}} J_{s_{3}}\right\rangle_{\text {odd }}\right)
$$

where $a$ and $b$ are independent constants, and the 'even' structure arises from free field theory.

The procedure, quite similar to that used by [17], is as follows. For any particular three point function we first consider the linearly independent basis of monomial structures (listed in section 7.3.1) and take an arbitrary linear combination of these structures.

$$
\left\langle J_{s_{1}} J_{s_{2}} J_{s_{3}}\right\rangle=\frac{1}{\tilde{X}_{12} \tilde{X}_{23} \tilde{X}_{31}} \sum_{n} a_{n} \mathcal{F}_{n}
$$

Current conservation $D_{\alpha_{1}} J^{\alpha_{1} \alpha_{2} \ldots \ldots \alpha_{2 s}}=0$ is tantamount to the following equation on the contracted current $J_{s}(x, \lambda)$ :

$$
D_{\alpha} \frac{\partial}{\partial \lambda_{\alpha}} J_{s}=0
$$

Thus the equation

$$
D_{i} \frac{\partial}{\partial \lambda_{i}}\left\langle J_{s_{1}} J_{s_{2}} J_{s_{3}}\right\rangle=0
$$

for each $i=1,2,3$ gives additional constraints in the form of linear equations in the $a_{n}$ 'ssome of these constants can thus be determined. The algebraic manipulations get quite unwieldy- we used superconformal invariance to set some co-ordinates to particular values and took recourse to Mathematica. The results obtained are given below (the known $\tilde{X}_{i j}$ dependent factors in the denominator are not listed below):

Using expression (4.12) for the currents in the $\mathcal{N}=1$ free theory, some 3-point functions were explicitly evaluated (again using Mathematica, $\mathrm{s}$ the computations get quite cumbersome beyond a few lower spin examples). It must be emphasized that the (tabulated) even structures obtained above match with the expressions obtained from free field theory (upto overall constants). We thus have some evidence for the claim that the threepoint function of conserved currents has a parity even part (generated by a free field theory) and a parity odd piece. 


\section{Summary and outlook}

In this paper we have embarked on the study of superconformal Chern-Simons matter theories in an on-shell superspace formalism. To conclude we summarize the main results obtained in this paper below.

- An explicit construction of higher spin conserved supercurrents in terms of higher spin component currents in section 4.1.

- An explicit construction of higher spin conserved supermultiplets in terms of on shell elementary superfields in free superconformal field theories in section 4.2.

- A decomposition of the state content of single trace operators in large $N$ vector Chern-Simons superconformal theories into multiplets of the superconformal algebra in the theories with $\mathcal{N}=1,2,3,4,6$ superconformal symmetry in appendix C.

- Determination of the form of two point functions of conserved higher spin supercurrents, and the explicit computation of these 2 point functions in free theories in section 6 .

- Classification of superconformal invariants formed out of 3 polarization spinors and 3 superspace insertion points (following [21]) and use thereof to constrain 3 point functions of higher spin operators in $3 \mathrm{~d}$ superconformal field theories in section 7.1.

- A conjecture - and evidence - that there are exactly two structure allowed in the 3 point functions of the conserved higher spin currents for $\mathcal{N}=1$ in section 7.3.2.

- The superspace structure of higher spin symmetry breaking on adding interactions to large $N$ gauge theories in section 5 .

One of the main motivations for the study embarked upon in this paper is to perform a Maldacena-Zhiboedov type study of superconformal Chern-Simons vector matter theories. As shown in the section (5), the structure of terms violating higher spin current conservation is much more constrained in superconformal case as compared to the conformal case suggesting that higher spin correlators in superconformal case must be more severely constrained. For this purpose it will be useful to extend the analysis of three-point functions presented here for $\mathcal{N}=1$ case to extended supersymmetry. Besides describing a variety of renormalization group fixed points in 3 dimensions, theories of this type are also expected to be holographic duals to supersymmetric higher spin Vasiliev theories in 4 dimensions. It may also be worth extending this formalism for 4 and higher point functions by using polarization spinor techniques, perhaps together with the embedding formalism, in view of implementing the (super)conformal bootstrap for higher spin operators.

\section{Acknowledgments}

We thank Prof. Shiraz Minwalla for suggesting this problem to us and for his guidance and helpful suggestions during the course of this project. We also thank R. Loganayagam and S. 
Prakash for useful discussions. We acknowledge the use of Matthew Headrick's grassmann Mathematica package for doing computations with fermionic variables. AAN thanks the Dept. of Theoretical Physics, TIFR, Mumbai and also the Crete Centre for Theoretical Physics, Heraklion (Erasmus IP in Non-perturbative QFT) for hospitality while this work was in progress. AAN would also like to thank Trinity College, Cambridge for a Rouse Ball Travelling Studentship and the Cambridge Commonwealth Trust for partial financial support. TS would like to thank ICTP, Trieste for hospitality while this work was in progress. Finally, TS and VU would like acknowledge our debt to the people of India for their generous and steady support to research in the basic sciences.

\section{A Conventions}

\section{A.1 Spacetime spinors}

The Lorentz group in $D=3$ is $\operatorname{SL}(2, \mathbb{R})$ (see, for instance, the appendix of [28]) and we can impose the Majorana condition on spinors, i.e., the fundamental representation is a real two component spinor $\psi_{\alpha}=\psi_{\alpha}^{*}(\alpha=1,2)$. The metric signature is mostly plus. $D=3$ superconformal theories with $\mathcal{N}$ extended supersymmetry posses an $\mathrm{SO}(\mathcal{N}) R$-symmetry which is part of the superconformal algebra, whose generators are real antisymmetric matrices $I^{a b}$, where $a, b$ are the vector indices of $\mathrm{SO}(\mathcal{N})$. The supercharges carry a vector $R$-symmetry index, $Q_{\alpha}^{a}$, as do the superconformal generators $S_{\alpha}^{a}$.

In $D=3$ we can choose a real basis for the $\gamma$ matrices

$$
\left(\gamma_{\mu}\right)_{\alpha}^{\beta} \equiv\left(i \sigma^{2}, \sigma^{1}, \sigma^{3}\right)=\left(\left(\begin{array}{rr}
0 & 1 \\
-1 & 0
\end{array}\right),\left(\begin{array}{cc}
0 & 1 \\
1 & 0
\end{array}\right),\left(\begin{array}{cc}
1 & 0 \\
0 & -1
\end{array}\right)\right)
$$

Gamma matrices with both indices up (or down) are symmetric

$$
\left(\gamma_{\mu}\right)_{\alpha \beta} \equiv\left(\mathbb{1}, \sigma^{3},-\sigma^{1}\right) \quad\left(\gamma_{\mu}\right)^{\alpha \beta} \equiv\left(\mathbb{1},-\sigma^{3}, \sigma^{1}\right)
$$

The antisymmetric $\epsilon$ symbol is $\epsilon^{12}=-1=\epsilon_{21}$. It satisfies

$$
\begin{aligned}
\epsilon \gamma^{\mu} \epsilon^{-1} & =-\left(\gamma^{\mu}\right)^{T} \\
\epsilon \Sigma^{\mu \nu} \epsilon^{-1} & =-\left(\Sigma^{\mu \nu}\right)^{T}
\end{aligned}
$$

where $\Sigma^{\mu \nu}=-\frac{i}{4}\left[\gamma^{\mu}, \gamma^{\nu}\right]$ are the Lorentz generators. The charge conjugation matrix $C$ can be chosen to be the identity, which we take to be

$$
-\epsilon \gamma^{0}=C^{-1} \quad \gamma^{0} \epsilon^{-1}=C
$$

$C^{\alpha \beta}$ denotes the inverse of $C_{\alpha \beta}$. Spinors transform as follows

$$
\psi_{\alpha}^{\prime} \rightarrow-\left(\Sigma_{\mu \nu}\right)_{\alpha}^{\beta} \psi_{\beta} .
$$

Spinors are naturally taken to have index structure down, i.e., $\psi_{\alpha}$. 
The raising and lowering conventions are

$$
\begin{aligned}
& \psi^{\beta}=\epsilon^{\beta \alpha} \psi_{\alpha} \\
& \psi_{\alpha}=\epsilon_{\alpha \beta} \psi^{\beta}
\end{aligned}
$$

There is now only one way to suppress contracted spinor indices,

$$
\psi \chi=\psi^{\alpha} \chi_{\alpha}
$$

and this leads to a sign when performing Hermitian conjugation

$$
(\psi \chi)^{*}=-\chi^{*} \psi^{*}
$$

The $\gamma$ matrices satisfy

$$
\left(\gamma_{\mu} \gamma_{\nu}\right)_{\alpha}^{\beta}=\eta_{\mu \nu} \delta_{\alpha}^{\beta}+\epsilon_{\mu \nu \rho}\left(\gamma^{\rho}\right)_{\alpha}^{\beta}
$$

where $\epsilon_{\mu \nu \rho}$ is the Levi-Civita symbol, and we set $\epsilon_{012}=1\left(\epsilon^{012}=-1\right)$. The superconformal algebra is given below:

$$
\begin{aligned}
{\left[M_{\mu \nu}, M_{\rho \lambda}\right] } & =i\left(\eta_{\mu \rho} M_{\nu \lambda}-\eta_{\nu \rho} M_{\mu \lambda}-\eta_{\mu \lambda} M_{\nu \rho}+\eta_{\nu \lambda} M_{\mu \rho}\right), \\
{\left[M_{\mu \nu}, P_{\lambda}\right] } & =i\left(\eta_{\mu \lambda} P_{\nu}-\eta_{\nu \lambda} P_{\mu}\right), \\
{\left[M_{\mu \nu}, K_{\lambda}\right] } & =i\left(\eta_{\mu \lambda} K_{\nu}-\eta_{\nu \lambda} K_{\mu}\right), \\
{\left[D, P_{\mu}\right] } & =i P_{\mu}, \quad\left[D, K_{\mu}\right]=-i K_{\mu}, \\
{\left[P_{\mu}, K_{\nu}\right] } & =2 i\left(\eta_{\mu \nu} D-M_{\mu \nu}\right), \\
{\left[I_{a b}, I_{c d}\right] } & =i\left(\delta_{a c} I_{b d}-\delta_{b c} I_{a d}-\delta_{a d} I_{b c}+\delta_{b d} I_{a c}\right), \\
\left\{Q_{\alpha}^{a}, Q_{\beta}^{b}\right\} & =\left(\gamma^{\mu}\right)_{\alpha \beta} P_{\mu} \delta^{a b}, \\
{\left[I_{a b}, Q_{c}^{\alpha}\right] } & =i\left(\delta_{a c} Q_{b}^{\alpha}-\delta_{b c} Q_{a}^{\alpha}\right), \\
\left\{S_{\alpha}^{a}, S_{\beta}^{b}\right\} & =\left(\gamma^{\mu}\right)_{\alpha \beta} K_{\mu} \delta^{a b} \\
{\left[I_{a b}, S_{c}^{\alpha}\right] } & =i\left(\delta_{a c} S_{b}^{\alpha}-\delta_{b c} S_{a}^{\alpha}\right), \\
{\left[K_{\mu}, Q_{\alpha}^{a}\right] } & =i\left(\gamma_{\mu}\right)_{\alpha}^{\beta} S_{\beta}^{a}, \\
{\left[P_{\mu}, S_{\alpha}^{a}\right] } & =i\left(\gamma_{\mu}\right)_{\alpha}^{\beta} Q_{\beta}^{a}, \\
{\left[D, Q_{\alpha}^{a}\right] } & =\frac{i}{2} Q_{\alpha}^{a}, \quad\left[D, S_{\alpha}^{a}\right]=-\frac{i}{2} S_{\alpha}^{a}, \\
{\left[M_{\mu \nu}, Q_{\alpha}^{a}\right] } & =-\left(\Sigma_{\mu \nu}\right)_{\alpha}^{\beta} Q_{\beta}^{a}, \\
{\left[M_{\mu \nu}, S_{\alpha}^{a}\right] } & =-\left(\Sigma_{\mu \nu}\right)_{\alpha}^{\beta} S_{\beta}^{a}, \\
\left\{Q_{\alpha}^{a}, S_{\beta}^{b}\right\} & =\left(\epsilon_{\beta \alpha} D-\frac{1}{2} \epsilon_{\mu \nu \rho}\left(\gamma^{\rho}\right)_{\alpha \beta} M^{\mu \nu}\right) \delta^{a b}+\epsilon_{\beta \alpha} I^{a b} .
\end{aligned}
$$

All other (anti)-commutators vanish.

\section{A.2 $R$-symmetry}

\section{A.2.1 $\mathrm{SO}(3)$}

Gamma matrices are chosen to be the sigma matrices

$$
\left(\sigma^{a}\right)_{i}^{j}=\left(\left(\begin{array}{ll}
0 & 1 \\
1 & 0
\end{array}\right),\left(\begin{array}{cc}
0 & -i \\
i & 0
\end{array}\right),\left(\begin{array}{cc}
1 & 0 \\
0 & -1
\end{array}\right)\right) .
$$


Indices are raised and lowered by $\epsilon^{12}=-1=-\epsilon_{12}$. Note that $\sigma$ matrices with both lower or both upper indices are symmetric.

The following identities are useful

$$
\begin{aligned}
\epsilon^{i j} \phi^{k} & +\epsilon^{j k} \phi^{i}+\epsilon^{k i} \phi^{j}=0, \\
\epsilon^{i j} \epsilon_{k l} & =\delta_{l}^{i} \delta_{k}^{j}-\delta_{k}^{i} \delta_{l}^{j}, \\
\epsilon_{i j} \epsilon_{k l} & =\epsilon_{i k} \epsilon_{j l}-\epsilon_{i l} \epsilon_{j k}, \quad \text { (same for upper indices) } \\
\left(\sigma^{a}\right)_{i}{ }^{j}\left(\sigma^{a}\right)_{k}{ }^{l} & =2 \delta_{i}^{l} \delta_{k}^{j}-\delta_{i}^{j} \delta_{k}^{l} \\
\left(\sigma^{a}\right)_{i j}\left(\sigma^{a}\right)_{k l} & =-\left(2 \epsilon_{i l} \epsilon_{j k}+\epsilon_{i j} \epsilon_{k l}\right)=-\left(\epsilon_{i k} \epsilon_{j l}+\epsilon_{i l} \epsilon_{j k}\right)
\end{aligned}
$$

\section{A.2.2 $\mathrm{SO}(4)$}

Gamma matrices are chosen to be

$$
\Gamma^{a}=\left(\begin{array}{ll}
0 & \sigma^{a} \\
\bar{\sigma}^{a} & 0
\end{array}\right) \text { for } a=1,2 \ldots 4
$$

where $\left(\sigma^{a}\right)_{i}^{\tilde{i}}=\left(\sigma^{1}, \sigma^{2}, \sigma^{3}, i \mathbb{1}_{2}\right), \quad\left(\bar{\sigma}^{a}\right)_{\tilde{i}}^{i}=\left(\sigma^{1}, \sigma^{2}, \sigma^{3},-i \mathbb{1}_{2}\right)$.

Indices are raised and lowered by $\epsilon^{12}=-\epsilon_{12}=-1=\tilde{\epsilon}^{12}=-\tilde{\epsilon}_{12}$. With these definitions, the following identities would be useful.

$$
\begin{aligned}
\left(\bar{\sigma}^{a}\right)^{\tilde{i} i} & =\left(\bar{\sigma}^{a T}\right)^{\tilde{i} i} \quad\left(\left(\bar{\sigma}^{a}\right)^{T}=-\epsilon \sigma^{a} \tilde{\epsilon}^{-1}\right), \\
\left(\sigma^{a}\right)_{i}{ }_{i}^{\tilde{i}}\left(\bar{\sigma}^{a}\right)_{\tilde{j}}^{j} & =2 \delta_{\tilde{j}}^{\tilde{i}} \delta_{i}^{j}, \\
\left(\sigma^{a}\right)^{\tilde{i}}\left(\bar{\sigma}^{a}\right)^{\tilde{j} j} & =-2 \epsilon^{i j} \epsilon^{\tilde{i} j}, \quad\left(\sigma^{a}\right)_{i \tilde{i}}\left(\bar{\sigma}^{a}\right)_{\tilde{j} j}=-2 \epsilon_{i j} \epsilon_{\tilde{i} \tilde{j}} .
\end{aligned}
$$

\section{A.2.3 $\mathrm{SO}(6)$}

We choose the gamma matrices to be

$$
\Gamma^{a}=\left(\begin{array}{ll}
0 & \gamma^{a} \\
\bar{\gamma}^{a} & 0
\end{array}\right) \text { for } a=1,2 \ldots 6
$$

where $\gamma^{a}=\left(\gamma^{1}, \gamma^{2}, \gamma^{3}, \gamma^{4}, \gamma^{5}, i \mathbb{1}_{4}\right), \bar{\gamma}^{a}=\left(\gamma^{1}, \gamma^{2}, \gamma^{3}, \gamma^{4}, \gamma^{5},-i \mathbb{1}_{4}\right), \gamma^{5}=\gamma^{1} \gamma^{2} \gamma^{3} \gamma^{4}$.

$$
\text { and } \gamma^{i}=\left(\begin{array}{cc}
0 & \sigma^{i} \\
\bar{\sigma}^{i} & 0
\end{array}\right) \text { with } \sigma^{i}=\left(\sigma^{1}, \sigma^{2}, \sigma^{3}, i \mathbb{1}_{2}\right), \bar{\sigma}^{i}=\left(\sigma^{1}, \sigma^{2}, \sigma^{3},-i \mathbb{1}_{2}\right) \text { for } i=1 \ldots 4
$$

In these basis we the 'chirality' projection matrix is diagonal and is given by

$$
\Gamma^{7}=-i \Gamma^{0} \Gamma^{1} \Gamma^{2} \Gamma^{3} \Gamma^{4} \Gamma^{5}=\left(\begin{array}{cc}
I_{4} & 0 \\
0 & -I_{4}
\end{array}\right)
$$

The charge conjugation matrix is

$$
C=\Gamma^{0} \Gamma^{2} \Gamma^{4}=\left(\begin{array}{cc}
0 & c \\
-c & 0
\end{array}\right) \text { with } c=i \gamma^{2} \gamma^{4}
$$


which satisfies

$$
\begin{aligned}
C^{*} & =C^{-1}=-C, \quad\left(\Gamma^{a}\right)^{*}=C^{-1} \Gamma^{a} C \\
\Rightarrow \quad c & =-c^{*}=c^{-1}, \quad\left(\bar{\gamma}^{a}\right)^{*}=-c^{-1} \gamma^{a} c
\end{aligned}
$$

In index notation: $\left(\bar{\gamma}_{i}^{a}{ }^{j}\right)^{*}=\left(\bar{\gamma}^{a *}\right)^{i}{ }_{j}=-c^{i k}\left(\gamma^{a}\right)_{k}{ }^{l} c_{l j}=c^{i k} c_{j l}\left(\gamma^{a}\right)_{k}{ }^{l}$

Indices are raised and lowered with using the charge conjugation matrix $C$ for $\Gamma^{a}$ and $c$ for $\gamma^{a}$. With both indices up or down the $\gamma$ matrices are antisymmetric. ${ }^{20}$ The last equation in (A.15) implies the following useful properties for the generators Let us define

$$
\gamma^{a b}=\gamma^{a} \bar{\gamma}^{b}-\gamma^{b} \bar{\gamma}^{a}, \quad \bar{\gamma}^{a b}=\bar{\gamma}^{a} \gamma^{b}-\bar{\gamma}^{b} \gamma^{a}
$$

then we have following useful relations

$$
\begin{aligned}
\gamma^{a b \dagger} & =-\gamma^{a b}, & \bar{\gamma}^{a b \dagger} & =-\bar{\gamma}^{a b}, \\
\left(\bar{\gamma}^{a b *}\right)_{j}^{i} & =\left(c^{-1} \gamma^{a b} c\right)^{i}{ }_{j}, & \left(\bar{\gamma}^{a b}\right)_{i}{ }^{j} & =-\left(c^{-1} \gamma^{a b} c\right)^{j}{ }_{i} .
\end{aligned}
$$

The first line says that the generators of $\mathrm{SO}(6)$ transformation are Hermitian ${ }^{21}$ while the two equation in the second line follows from (A.15).

The following identities are useful: ${ }^{22}$

$$
\begin{aligned}
\bar{\gamma}_{i j}^{a} & =\gamma_{i j}^{a}+2 \delta^{a 0} c_{i j}, \quad\left(\bar{\gamma}^{a}\right)_{i}^{j}=\left(\gamma^{a}\right)_{i}^{j}-2 \delta^{a 0} \delta_{i}^{j}, \\
\gamma_{i j}^{a} \gamma_{k l}^{a} & =-2 \epsilon_{i j k l}=2\left(c_{i k} c_{j l}-c_{i l} c_{j k}-c_{i j} c_{k l}\right), \\
\gamma_{i j}^{a} \bar{\gamma}_{k l}^{a} & =-2 \epsilon_{i j k l}+2 c_{i j} c_{k l}=2\left(c_{i k} c_{j l}-c_{i l} c_{j k}\right), \\
\left(\gamma^{a}\right)^{i j}\left(\bar{\gamma}^{a}\right)_{k l} & =2 \delta_{k}^{i} \delta_{l}^{j}-2 \delta_{l}^{i} \delta_{k}^{j}, \\
\left(\gamma^{a b}\right)_{i}^{j}\left(\gamma^{a b}\right)_{k}^{l} & =-32 \delta_{i}^{l} \delta_{k}^{j}+8 \delta_{i}^{j} \delta_{k}^{l},
\end{aligned}
$$

\section{A.3 Useful relations}

Some useful relations and identities are given below

$$
\begin{array}{rlrl}
\epsilon^{\alpha \beta} \frac{\partial}{\partial \theta^{a \beta}} & =-\frac{\partial}{\partial \theta_{\alpha}^{a}} & \\
\left(\gamma^{\mu}\right)_{\alpha}^{\beta}\left(\gamma_{\mu}\right)_{\sigma}{ }^{\rho} & =2 \delta_{\alpha}{ }^{\beta} \delta_{\sigma}^{\beta}-\delta_{\alpha}{ }^{\beta} \delta_{\sigma}{ }^{2} & \\
\theta_{\alpha} \theta_{\beta} & =\frac{1}{2} \epsilon_{\alpha \beta} \theta \theta, & & \theta^{\alpha} \theta^{\beta}=-\frac{1}{2} \epsilon^{\alpha \beta} \theta \theta \\
\theta_{1 \alpha} \theta_{2}^{\beta}+\theta_{2 \alpha} \theta_{1}^{\beta}+\left(\theta_{1} \theta_{2}\right) \delta_{\alpha}^{\beta} & =0 & \\
X^{2} \equiv X_{\alpha}{ }^{\beta} X_{\beta}{ }^{\alpha}=2 x_{\mu} x^{\mu} & \equiv 2 x^{2}, & X_{\alpha}^{\beta} X_{\beta}^{\gamma}=x^{2} \delta_{\alpha}^{\gamma}=\frac{X^{2}}{2}
\end{array}
$$

\footnotetext{
${ }^{20}$ This should be the case as the vector of $\mathrm{SO}(6)$ is $(4 \times 4)$ antisym of $\mathrm{SU}(4)$.

${ }^{21}$ The generator of $\mathrm{SO}(6)$ acting on chiral and antichiral transformation are respectively $-\frac{i}{4} \gamma^{a b}$ and $-\frac{i}{4} \bar{\gamma}^{a b}$.

${ }^{42}$ Note that representation theory (SU(4)) wise $C$ shouldn't be used to raise or lower indices as it is not an invariant tensor of SU(4). Only $\epsilon^{i j k l}$ and $\epsilon_{i j k l}$ (which are specific combinations of product of c's) can be used to raise or lower SU(4) indices. we will explicitly see that all the SU(4) tensor equations can be written using just $\epsilon$ tensors.
} 


$$
\begin{aligned}
D_{1 \alpha} \tilde{X}_{12}^{-\Delta} & =i \Delta\left(\tilde{X}_{12}\right)_{\alpha}^{\beta}\left(\theta_{12}\right)_{\beta} & \\
D_{1 \alpha}\left(\tilde{X}_{12}\right)_{\beta}^{\gamma} & =-i \delta_{\alpha}^{\gamma}\left(\theta_{12}\right)_{\beta}+\frac{i}{2} \delta_{\beta}^{\gamma}\left(\theta_{12}\right)_{\alpha} & \\
D_{1 \alpha}\left(X_{12-}\right)_{\beta}^{\gamma} & =-i \delta_{\alpha}^{\gamma} \theta_{12 \beta}, & D_{1 \alpha}\left(X_{12+}\right)_{\beta}^{\gamma}=i \epsilon_{\alpha \beta} \theta_{12}^{\gamma}
\end{aligned}
$$

\section{B Conformal spectrum of free scalars and fermions}

In this appendix we list the character decomposition of product of two short conformal representations into irreducible conformal representations for the particular cases of a complex scalar and complex fermions. Let us denote by $\chi(\phi)$ and $\chi(\psi)$ the conformal character of a free scalar field and a free fermion (in $D=3$ ) respectively. Then we have

$$
\begin{gathered}
\chi(\phi) \chi(\bar{\phi})=\frac{1}{(1-x)(1-x y)\left(1-x y^{-1}\right)}\left(x+\sum_{k=1}^{\infty} \chi_{(s h)}(k+1, k)\right) \\
\chi(\bar{\phi}) \chi(\psi)=\chi(\phi) \chi(\bar{\psi})=\frac{1}{(1-x)(1-x y)\left(1-x y^{-1}\right)}\left(x^{\frac{3}{2}} \chi_{\frac{1}{2}}(y)+\sum_{k=1}^{\infty} \chi_{(s h)}\left(k+\frac{3}{2}, k+\frac{1}{2}\right)\right) \\
\chi(\psi) \chi(\bar{\psi})=\frac{1}{(1-x)(1-x y)\left(1-x y^{-1}\right)}\left(x^{2}+\sum_{k=1}^{\infty} \chi_{(s h)}(k+1, k)\right)
\end{gathered}
$$

where the $\chi_{(s h)}(j+1, j)$ denotes the character of a short conformal representation with spin $j$.

Let us consider a free $\mathcal{N}=1$ superconformal theory of a complex boson and a complex fermion transforming in $N$ of $\mathrm{SU}(N)$ gauge group. The spectrum of gauge invariant single trace operators in theory is then just the sum of the operators represented in (B.1). Using the decomposition in (B.3), the operators in (B.1) are easily combined into the representation of $\mathcal{N}=1$ supermultiplets. These are given as follows ${ }^{23}$

$$
\left(\frac{1}{2}, 0\right)_{1} \oplus \sum_{k=1}^{\infty}\left(\frac{k}{2}+1, \frac{k}{2}\right)_{1}
$$

i.e. along with the special short representation with spin zero there are superconformal short representation for every positive half integer spin starting from spin $\frac{1}{2}$. For convenience we list the decomposition of all short and long $\mathcal{N}=1$ superconformal representations below

$$
\begin{aligned}
(\Delta, j)_{1, \text { long }} & =(\Delta, j) \oplus\left(\Delta+\frac{1}{2}, j-\frac{1}{2}\right) \oplus\left(\Delta+\frac{1}{2}, j+\frac{1}{2}\right) \oplus(\Delta+1, j), \\
(j+1, j)_{1} & =(j+1, j) \oplus\left(j+\frac{3}{2}, j+\frac{1}{2}\right), \\
\left(\frac{1}{2}, 0\right)_{1} & =\left(\frac{1}{2}, 0\right) \oplus\left(1, \frac{1}{2}\right) \oplus\left(\frac{3}{2}, 0\right) .
\end{aligned}
$$

\footnotetext{
${ }^{23}$ Here $(\Delta, j)_{1}$ denote $\mathcal{N}=1$ representation while $(\Delta, j)$ denotes a conformal representation.
} 


\section{Superconformal spectrum of $\mathcal{N}=1,2,3,4,6$ theories}

In this appendix we discuss the full single trace gauge invariant local operator spectrum of the free $\mathrm{U}(N)$ superconformal Chern-Simons vector theories discussed in section 3. In subsequent subsections here we present the full conformal primary spectrum, using the conformal grouping discussed in appendix B, and then group these conformal primaries into representations of superconformal algebra of the respective theory. ${ }^{24}$

\section{C.1 $\mathcal{N}=1$}

The minimal field content of this theory consists of a complex scalar and a complex fermion. The conformal content is easy to write down; there are both integer and half-integer spin currents in the theory. All of these group into short superconformal multiplets of both integer and half integer spin. Thus, the superconformal primary content of this theory is

$$
\bigoplus_{j=0, \frac{1}{2}, 1, \ldots}^{\infty}(j+1, j)_{\mathcal{N}=1},
$$

where $(j+1, j)$ denotes a dimension $j+1$, spin $j$ short superconformal primary multiplet which contains the conserved spin $j$ and spin $j+\frac{1}{2}$ conformal primaries. There is no $R$-symmetry quantum number in this case. The conformal content is

$$
\begin{array}{rlrl}
(j+1, j)_{\mathcal{N}=1} & \rightarrow(j+1, j) \oplus\left(j+\frac{3}{2}, j+\frac{1}{2}\right) & j \neq 0 \\
(1,0)_{\mathcal{N}=1} \rightarrow(1,0) \oplus\left(\frac{3}{2}, \frac{1}{2}\right) \oplus(2,0) & j=0 .
\end{array}
$$

\section{C.2 $\mathcal{N}=2$}

The field content of the $\mathcal{N}=2$ theories is the same as that of $\mathcal{N}=1$; the difference being that the spectrum of short superconformal multiplets consists only of integer spins. Thus, we can write the spectrum of short superconformal primaries in these theories as

$$
\bigoplus_{j=0,1, \ldots}^{\infty}(j+1, j, 0)_{\mathcal{N}=2}
$$

The conformal content for a spin $j \mathcal{N}=2$ short superconformal primary in terms of $\mathcal{N}=1$ is

$$
(j+1, j, 0)_{\mathcal{N}=2} \rightarrow(j+1, j)_{\mathcal{N}=1} \oplus\left(j+\frac{3}{2}, j+\frac{1}{2}\right)_{\mathcal{N}=1}
$$

from which he conformal content can be read off as

$$
\begin{array}{rlrl}
(j+1, j, 0)_{\mathcal{N}=2} & \rightarrow(j+1, j, 0) \oplus\left(j+\frac{3}{2}, j+\frac{1}{2}, 1\right) \oplus\left(j+\frac{3}{2}, j+\frac{1}{2},-1\right) \oplus(j+2, j+1,0) & j \neq 0 \\
(1,0,0)_{\mathcal{N}=2} \rightarrow(1,0,0) \oplus\left(\frac{3}{2}, \frac{1}{2}, 1\right) \oplus(2,0,0) \oplus\left(\frac{3}{2}, \frac{1}{2},-1\right) \oplus(2,1,0) & j=0 .
\end{array}
$$

where the third quantum number is the $\mathrm{U}(1)_{R}$ charge.

\footnotetext{
${ }^{24}$ Representation of superconformal algebra are labeled by the scaling dimension, spin and R-symmetry representation of the superconformal primary. see e.g. section 3 of [29] a summary of unitary representations of superconformal algebra in $2+1 \mathrm{~d}$.
} 


\section{C.3 $\mathcal{N}=3$}

The conformal content of the $\mathcal{N}=3$ theory is ${ }^{25}$

$$
\left.\left.2 \bigoplus_{j=0, \frac{1}{2}, 1, \ldots}^{\infty}[(j+1, j, 1) \oplus(j+1, j, 3)]\right] \oplus(1,0,1) \oplus(1,0,3) \oplus(2,0,1) \oplus(2,0,3) \quad \text { (C. } 6\right)
$$

The above conformal content can be grouped into $\mathcal{N}=3$ superconformal primary content as follows

$$
\left[\bigoplus_{j=0, \frac{1}{2}, 1, \ldots}^{\infty}(j+1, j, 1)_{\mathcal{N}=3}\right] \oplus(1,0,3)_{\mathcal{N}=3}
$$

The decomposition of the $\mathcal{N}=3$ superconformal primaries into $\mathcal{N}=2$ superconformal primaries, given in [29] is ${ }^{26}$

$$
(j+1, j, 1)_{\mathcal{N}=3} \longrightarrow(j+1, j, 0)_{\mathcal{N}=2} \oplus\left(j+\frac{3}{2}, j+\frac{1}{2}, 0\right)_{\mathcal{N}=2}
$$

We have the following result for the conformal content of a $\mathcal{N}=3$ superconformal primary of spin $j \in\left(0, \frac{1}{2}, 1, \ldots\right)$ :

$$
(j+1, j, 1)_{\mathcal{N}=3} \rightarrow(j+1, j, 1) \oplus\left(j+\frac{3}{2}, j+\frac{1}{2}, 3\right) \oplus(j+2, j+1,3) \oplus\left(j+\frac{5}{2}, j+\frac{3}{2}, 1\right)
$$

The breakup of the $(1,0,3)_{\mathcal{N}=3}$ superconformal primary into $\mathcal{N}=2$ primaries is as follows ${ }^{18}$

$$
(1,0,3)_{\mathcal{N}=3} \rightarrow(1,0,1)_{\mathcal{N}=2} \oplus(1,0,0)_{\mathcal{N}=2} \oplus(1,0,-1)_{\mathcal{N}=2}
$$

The conformal content of the $(1,0,3)_{\mathcal{N}=3}$ superconformal primary is: ${ }^{27}$

$$
(1,0,3)_{\mathcal{N}=3} \rightarrow(1,0,3) \oplus(2,0,3) \oplus\left(\frac{3}{2}, \frac{1}{2}, 1\right) \oplus\left(\frac{3}{2}, \frac{1}{2}, 3\right) \oplus(2,1,1)
$$

\section{C.4 $\mathcal{N}=4$}

The theory contains currents of integer spins only. It remains to comment about the $(1,0,3)$ superconformal primary which was obtained in equation (C.7) above. This particular primary transforms in the antisymmetric $(1,0)$ representation of the $\mathrm{SO}(4) \sim \mathrm{SU}(2) \times \mathrm{SU}(2) R$ symmetry, where the two numbers correspond to each of the two SU(2)s. Therefore we have

$$
\left[\bigoplus_{j=0,1, \ldots}^{\infty}(j+1, j,\{0,0\})_{\mathcal{N}=4}\right] \oplus(1,0,\{1,0\})_{\mathcal{N}=4}
$$

where by $\{0,0\}$ we mean the singlet of the $\mathrm{SU}(2) \times \mathrm{SU}(2) R$-symmetry.

\footnotetext{
${ }^{25}$ For $R$-symmetry quantum numbers taking values in $\mathrm{SU}(2)_{R}$, we give the dimension of the representation while writing down the quantum numbers $(\Delta, j, h)$. For example, $(1,0,1)$ corresponds to $\Delta=1$, spin- 0 and a singlet under $R$. In other words, instead of writing the highest weight $j$ for the $R$-symmetry representation, we write $2 j+1$ as the third quantum number.

${ }^{26}$ In the equation that follows note that the l.h.s. is written in terms of the $\mathrm{SU}(2)_{R}$ quantum number whereas the r.h.s. has $\mathrm{U}(1)_{R}$ quantum numbers.

${ }^{27}$ Written out in $\mathrm{SU}(2)_{R}$ notation.
} 


\section{C.5 $\mathcal{N}=6$}

From the field content of the $\mathcal{N}=6$ theory (see section 3.5) the conformal primary spectrum can be easily read off as

$$
\begin{aligned}
& {\left[2 \bigoplus_{j=0,1, \ldots}^{\infty}\left((j+2, j+1 ; 1) \oplus(j+2, j+1 ; 15) \oplus\left(j+\frac{3}{2}, j+\frac{1}{2} ; 6\right) \oplus\left(j+\frac{3}{2}, j+\frac{1}{2} ; 10\right)\right)\right]} \\
& \oplus(1,0 ; 1) \oplus(1,0 ; 15) \oplus(2,0 ; 1) \oplus(2,0 ; 15) \oplus
\end{aligned}
$$

where the conformal primaries are labeled as $(\Delta, j ; \mathrm{SO}(6)$ representation). In specifying the $\mathrm{SO}(6) R$-symmetry representation we use the following notation

$$
\begin{aligned}
1 & \rightarrow \text { Singlet, } \\
6 & \rightarrow \text { Vector, } \\
15 & \rightarrow \text { Second rank symmetric traceless tensor, } \\
10 & \rightarrow \text { (anti)Self-dual } 3 \text { form. }
\end{aligned}
$$

The conformal primary spectrum can be grouped together into the following $\mathcal{N}=6$ superconformal primary spectrum

$$
\left[\bigoplus_{j=1,2, \ldots}^{\infty}(j+1, j ; 1)_{\mathcal{N}=6}\right] \oplus(1,0 ; 15)_{\mathcal{N}=6}
$$

where again use the same labeling for the superconformal primary as above for conformal primaries with and extra subscript to distinguish from conformal primaries.

Open Access. This article is distributed under the terms of the Creative Commons Attribution License (CC-BY 4.0), which permits any use, distribution and reproduction in any medium, provided the original author(s) and source are credited.

\section{References}

[1] O. Aharony, G. Gur-Ari and R. Yacoby, $D=3$ bosonic vector models coupled to Chern-Simons gauge theories, JHEP 03 (2012) 037 [arXiv:1110.4382] [INSPIRE].

[2] S. Giombi et al., Chern-Simons theory with vector fermion matter, Eur. Phys. J. C 72 (2012) 2112 [arXiv:1110.4386] [INSPIRE].

[3] J. Maldacena and A. Zhiboedov, Constraining conformal field theories with a higher spin symmetry, J. Phys. A 46 (2013) 214011 [arXiv:1112.1016] [INSPIRE].

[4] J. Maldacena and A. Zhiboedov, Constraining conformal field theories with a slightly broken higher spin symmetry, Class. Quant. Grav. 30 (2013) 104003 [arXiv:1204.3882] [INSPIRE].

[5] S. Banerjee, S. Hellerman, J. Maltz and S.H. Shenker, Light states in Chern-Simons theory coupled to fundamental matter, JHEP 03 (2013) 097 [arXiv: 1207.4195] [INSPIRE].

[6] C.-M. Chang, S. Minwalla, T. Sharma and X. Yin, ABJ triality: from higher spin fields to strings, J. Phys. A 46 (2013) 214009 [arXiv:1207.4485] [InSPIRE]. 
[7] O. Aharony, G. Gur-Ari and R. Yacoby, Correlation functions of large- $N$ Chern-Simons-matter theories and bosonization in three dimensions, JHEP 12 (2012) 028 [arXiv: 1207.4593] [INSPIRE].

[8] S. Jain, S.P. Trivedi, S.R. Wadia and S. Yokoyama, Supersymmetric Chern-Simons theories with vector matter, JHEP 10 (2012) 194 [arXiv:1207.4750] [INSPIRE].

[9] S. Giombi and X. Yin, The higher spin/vector model duality, J. Phys. A 46 (2013) 214003 [arXiv:1208.4036] [INSPIRE].

[10] S. Banerjee et al., Smoothed transitions in higher spin AdS gravity, Class. Quant. Grav. 30 (2013) 104001 [arXiv:1209.5396] [INSPIRE].

[11] S. Yokoyama, Chern-Simons-fermion vector model with chemical potential, JHEP 01 (2013) 052 [arXiv: 1210.4109] [INSPIRE].

[12] G. Gur-Ari and R. Yacoby, Correlators of large-N fermionic Chern-Simons vector models, JHEP 02 (2013) 150 [arXiv:1211.1866] [INSPIRE].

[13] O. Aharony, S. Giombi, G. Gur-Ari, J. Maldacena and R. Yacoby, The thermal free energy in large-N Chern-Simons-matter theories, JHEP 03 (2013) 121 [arXiv:1211.4843] [INSPIRE].

[14] S. Jain et al., Phases of large- $N$ vector Chern-Simons theories on $S^{2} \times S^{1}$, JHEP 09 (2013) 009 [arXiv:1301.6169] [INSPIRE].

[15] T. Takimi, Duality and higher temperature phases of large- $N$ Chern-Simons matter theories on $S^{2} \times S^{1}$, JHEP 07 (2013) 177 [arXiv: 1304.3725] [INSPIRE].

[16] S. Jain, S. Minwalla and S. Yokoyama, Chern-Simons duality with a fundamental boson and fermion, JHEP 11 (2013) 037 [arXiv: 1305.7235] [INSPIRE].

[17] S. Giombi, S. Prakash and X. Yin, A note on CFT correlators in three dimensions, JHEP 07 (2013) 105 [arXiv: 1104.4317] [INSPIRE].

[18] J.-H. Park, $N=1$ superconformal symmetry in four-dimensions, Int. J. Mod. Phys. A 13 (1998) 1743 [hep-th/9703191] [InSPIRE].

[19] H. Osborn, $N=1$ superconformal symmetry in four-dimensional quantum field theory, Annals Phys. 272 (1999) 243 [hep-th/9808041] [INSPIRE].

[20] J.-H. Park, Superconformal symmetry in six-dimensions and its reduction to four-dimensions, Nucl. Phys. B 539 (1999) 599 [hep-th/9807186] [INSPIRE].

[21] J.-H. Park, Superconformal symmetry in three-dimensions, J. Math. Phys. 41 (2000) 7129 [hep-th/9910199] [INSPIRE].

[22] J.-H. Park, Superconformal symmetry and correlation functions, Nucl. Phys. B 559 (1999) 455 [hep-th/9903230] [INSPIRE].

[23] M.S. Costa, J. Penedones, D. Poland and S. Rychkov, Spinning conformal correlators, JHEP 11 (2011) 071 [arXiv:1107.3554] [INSPIRE].

[24] S.M. Kuzenko, J.-H. Park, G. Tartaglino-Mazzucchelli and R. Unge, Off-shell superconformal nonlinear $\sigma$-models in three dimensions, JHEP 01 (2011) 146 [arXiv:1011.5727] [INSPIRE].

[25] S.M. Kuzenko, U. Lindström and G. Tartaglino-Mazzucchelli, Off-shell supergravity-matter couplings in three dimensions, JHEP 03 (2011) 120 [arXiv:1101.4013] [INSPIRE].

[26] S. Banerjee and D. Radicevic, Chern-Simons theory coupled to bifundamental scalars, arXiv: 1308.2077 [INSPIRE]. 
[27] S.M. Kuzenko and S. Theisen, Correlation functions of conserved currents in $N=2$ superconformal theory, Class. Quant. Grav. 17 (2000) 665 [hep-th/9907107] [INSPIRE].

[28] T.T. Dumitrescu and N. Seiberg, Supercurrents and brane currents in diverse dimensions, JHEP 07 (2011) 095 [arXiv: 1106.0031] [INSPIRE].

[29] S. Minwalla, P. Narayan, T. Sharma, V. Umesh and X. Yin, Supersymmetric states in large-N Chern-Simons-matter theories, JHEP 02 (2012) 022 [arXiv:1104.0680] [INSPIRE]. 